ESAIM: M2AN 47 (2013) 281-304

DOI: $10.1051 / \mathrm{m} 2 \mathrm{an} / 2012028$
ESAIM: Mathematical Modelling and Numerical Analysis

www.esaim-m2an.org

\title{
ERROR ESTIMATES FOR A FITZHUGH-NAGUMO PARAMETER-DEPENDENT REACTION-DIFFUSION SYSTEM
}

\author{
Konstantinos Chrysafinos ${ }^{1}$, Sotirios P. Filopoulos ${ }^{2}$ \\ and Theodosios K. Papathanasiou ${ }^{2}$
}

\begin{abstract}
Space-time approximations of the FitzHugh-Nagumo system of coupled semi-linear parabolic PDEs are examined. The schemes under consideration are discontinuous in time but conforming in space and of arbitrary order. Stability estimates are presented in the natural energy norms and at arbitrary times, under minimal regularity assumptions. Space-time error estimates of arbitrary order are derived, provided that the natural parabolic regularity is present. Various physical parameters appearing in the model are tracked and numerical examples are presented.
\end{abstract}

Mathematics Subject Classification. 65M60, 35Q92, 92-08.

Received July 22, 2011. Revised April 26, 2012

Published online November 23, 2012.

\section{INTRODUCTION}

The FitzHugh-Nagumo (FHN) system consists of two parabolic PDEs which are coupled through nonlinear terms,

$$
\begin{aligned}
& \left\{\begin{aligned}
u_{t}-\Delta u+u^{3}-u & =-v+f_{1} & & \text { in }(0, T) \times \Omega \\
u & =0 & & \text { on }(0, T) \times \Gamma \\
u(0, x) & =u_{0} & & \text { in } \Omega,
\end{aligned}\right. \\
& \left\{\begin{aligned}
v_{t}-\delta \Delta v & =\epsilon\left(u-\alpha_{1} v\right)+f_{2} & & \text { in }(0, T) \times \Omega \\
v & =0 & & \text { on }(0, T) \times \Gamma \\
v(0, x) & =v_{0} & & \text { in } \Omega .
\end{aligned}\right.
\end{aligned}
$$

Here, $\Omega$ denotes a bounded domain in $\mathbb{R}^{2}$, with Lipschitz boundary $\Gamma, u_{0}, v_{0}$ and $f_{1}, f_{2}$ denote initial data and forcing terms respectively. The parameters $\epsilon, \delta, \alpha_{1}$ appearing in the model problem, represent different scales of the physical variables $u, v$ involved in our model. The FitzHugh-Nagumo equations were proposed for the modelling of the transmission of electrical impulses in a nerve axon (see e.g. $[19,26,35,36]$ ). They belong to a

\footnotetext{
Keywords and phrases. Error estimates, discontinuous time-stepping Galerkin schemes, FitzHugh-Nagumo equations, reactiondiffusion, parameter dependent, coarse time-stepping.

1 Department of Mathematics, National Technical University of Athnens, Zografou Campus, 15780 Athens, Greece. chrysafinos@math.ntua.gr

2 Section of Applied and Theoretical Mechanics, National Technical University of Athens, Zografou Campus, 15780 Athens,

Greece. sfilop@gmail.com; papathth@gmail.com
} 
general category of parabolic PDE systems of reaction-diffusion equations that model physical phenomena in biology (see e.g. $[18,26,36,38,42]$ ) of the form,

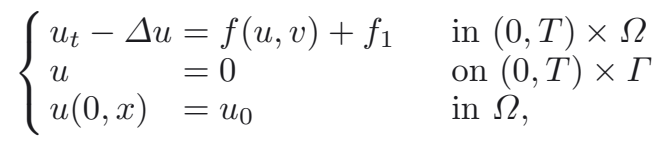

$$
\begin{aligned}
& \left\{\begin{aligned}
v_{t}-\delta \Delta v & =\epsilon g(u, v)+f_{2} & & \text { in }(0, T) \times \Omega \\
v & =0 & & \text { on }(0, T) \times \Gamma \\
v(0, x) & =v_{0} & & \text { in } \Omega .
\end{aligned}\right.
\end{aligned}
$$

The classical FitzHugh-Nagumo model is deduced from the above system by choosing $f(u, v)=u-u^{3}-v$, $g(u, v)=u-\alpha_{1} v$, and can be viewed as a simplified Hodgkin-Huxley model (see e.g. [26,36] and references within). These systems are coupled and parameter-dependent, hence they exhibit interesting dynamical behavior. For several results regarding mathematical analysis, including dynamics, of such systems we refer the reader to the books $[18,38]$ (see also references within).

Our main goal is to provide a rigorous analysis of a general class of numerical schemes that can be useful to applied scientists in studying the behavior of such systems. The main difficulties associated to the approximation of the FHN system, as well as to other similar reaction-diffusion parameter dependent systems, can be summarized as follows:

- Structural difficulties: the FHN system contains two parabolic PDEs which are coupled through nonlinear terms. In addition, the physical phenomena modeled by the above system posses complex dynamics for realistic values of the parameters $\epsilon, \delta$. In particular, the natural energy norms imposed by the structure of our problem scale differently in terms of these parameters, creating substantial difficulties in the analysis and computations of such problems.

- Regularity restrictions: the regularity of solutions $u, v$ is typically low, hence standard techniques, which require the presence of higher regularity, typically fail and/or require restrictions on the size of the timediscretization parameter $\tau$ in terms of the spatial discretization parameter $h$ that are prohibitively expensive for realistic computations. Even when enhanced parabolic regularity is present, due to standard boot-strap regularity arguments, it is not clear how to utilize high-order methods in order to speed up convergence of discrete schemes.

- Parameter dependence: the physical parameters $\epsilon, \delta$ should be tracked in the estimates since they effectively determine the size of various norms, and hence, the overall behavior of the system. In particular, great care should be exercised in order to avoid any exponential dependence of the time-step restriction to quantities of the form $1 / \epsilon$ or $1 / \delta$.

The schemes considered here are discontinuous (in time) but conforming in space. The motivation for using the discontinuous (in time) Galerkin approach relies to its performance in a vast area of problems with solutions of low regularity. In addition, as we will subsequently show discontinuous Galerkin schemes also provide the natural framework to explore coarse time-stepping approaches. The main feature of discontinuous time stepping Galerkin schemes is their ability to mimic the stability properties of the corresponding continuous system. In particular, we will show that such numerical schemes are stable under minimal regularity assumptions on the given data, i.e., $f_{1}, f_{2} \in L^{2}\left[0, T ; H^{-1}(\Omega)\right], u_{0}, v_{0} \in L^{2}(\Omega)$ in the natural energy norm and at arbitrary time-points.

The main goal of this work is to derive stability and error estimates of arbitrary order for which $\tau$ and $h$ can be chosen independently. More specifically, we demonstrate convergence under minimal regularity assumptions on the given data, and we derive optimal error estimates of order $\mathcal{O}\left(\tau^{k+1}+h^{l}\right)$ when solutions are sufficiently smooth. The above estimates are also applicable in case that standard conforming linear finite elements in space are combined with high order discontinuous time-stepping schemes. In addition, great care is exercised in order to track the appearance of the parameters $\delta, \epsilon, \alpha_{1}$ into various constants appearing in the estimates. 
Our computational results highlight the fact that discontinuous Galerkin time-stepping schemes provide a unified framework to treat both high and low order schemes. Numerical experiments also verify our theoretical findings, and in particular, the applicability of "coarse" time stepping approaches, where a few time-steps are needed in order to achieve the spatial convergence rate. Our technique follows the recently developed analysis of discontinuous time-stepping schemes for the Navier-Stokes equations [7] and can be extended in other reactiondiffusion parameter dependent systems.

Semi-discrete estimates for the FitzHugh-Nagumo equations have been analyzed in [29], when $\delta=0$, while error estimates for semi-discrete approximations for other reaction-diffusion parameter dependent systems have been recently obtained in [21,22]. Fully-discrete schemes (first-order in time) were considered in $[21,22,24]$, for other parameter dependent systems of reaction-diffusion equations. In particular, in [24] a first order scheme is analyzed under minimal regularity assumptions on the data for the forced Fisher equation, while in [21,22], a semi-implicit first order (in time) scheme is discussed for reaction-diffusion parameter dependent systems modelling predator-prey interactions.

For a system of hysteric reaction-diffusion equations, an alternating direction method is studied in [4], while convergence of order two is demonstrated for reaction-diffusion (parameter dependent) system using the Peaceman-Rachford approximation in [10]. Operator splitting techniques are also considered in the work of $[25]$.

For methods related to the numerical analysis of general semilinear parabolic PDEs we refer the reader to [40] and the references within. In [40] a survey of several results regarding a-priori and a-posteriori analysis of semilinear PDEs is presented for smooth solutions. An imlpicit-explicit multistep method for approximations of semi-linear parabolic PDEs is analyzed in the work of [3] and while linear implicit schemes were studied in [1]. The discontinuous (in time) Galerkin technique is analyzed in the works of $[2,9,11-15,30-32]$ for linear and semilinear problems. Several results regarding a posteriori error estimation of reaction-diffusion systems are presented in [16] (see also references within), while space-time adaptivity techniques for reaction-diffusion type of systems (including examples for the FHN system) are proposed in [20]. A survey of various numerical techniques associated to the methods of lines, Runge-Kutta and operator splitting methods is presented in [27] (see also references within). Finally, in the very recent work of [34], numerical techniques for the solution of optimal control problems related to FHN systems are analyzed.

An outline of this paper follows. After presenting some preliminaries in Section 2, the setup of the fully discrete problem, and the basic stability estimates of our FHN system under minimal regularity assumptions are presented in Section 3. These results are necessary in order to derive the error estimates of Section 4. Finally, in Section 5, we close this paper by presenting computational examples using high and low order in time schemes for smooth solutions, as well as a numerical example under minimal regularity assumptions.

\section{Preliminaries}

\subsection{Notation}

For any banach space $X$ we denote the time-space spaces by $L^{p}[0, T ; X], L^{\infty}[0, T ; X]$, endowed with norms:

$$
\|w\|_{L^{p}[0, T ; X]}=\left(\int_{0}^{T}\|w\|_{X}^{p} \mathrm{~d} t\right)^{\frac{1}{p}}, \quad\|w\|_{L^{\infty}[0, T ; X]}=\operatorname{esssup}_{t \in[0, T]}\|w\|_{X} .
$$

The set of all continuous functions $v:[0, T] \rightarrow X$, is denoted by $C[0, T ; X]$ endowed with norm

$$
\|w\|_{C[0, T ; X]}=\max _{t \in[0, T]}\|w(t)\|_{X} .
$$

For the definition of spaces $H^{s}[0, T ; X]$, we refer the reader to [17,42]. Typically, $X \equiv H^{s}(\Omega), 0<s \in \mathbb{R}$, where $H^{s}(\Omega)$ denotes the standard Sobolev (Hilbert) spaces. We denote by $H_{0}^{1}(\Omega) \equiv\left\{w \in H^{1}(\Omega):\left.w\right|_{\Gamma}=0\right\}$, and by $H^{-1}(\Omega)$ its dual. Finally, we use the notation $\langle.,$.$\rangle for the duality pairing of H^{-1}(\Omega), H_{0}^{1}(\Omega)$ and $(.,$.$) for$ 
the standard $L^{2}$ inner product. In the sequel, we will use the following (natural energy) space for the variables $u, v \in W(0, T)$ where,

$$
W(0, T)=L^{2}\left[0, T ; H_{0}^{1}(\Omega)\right] \cap H^{1}\left[0, T ; H^{-1}(\Omega)\right]
$$

with norm

$$
\|w\|_{W(0, T)}^{2}=\|w\|_{L^{2}\left[0, T ; H_{0}^{1}(\Omega)\right]}^{2}+\left\|w_{t}\right\|_{L^{2}\left[0, T ; H^{-1}(\Omega)\right]}^{2} .
$$

The bilinear form associated to our problem is defined by

$$
a\left(w_{1}, w_{2}\right)=\int_{\Omega} \nabla w_{1} \nabla w_{2} \mathrm{~d} x \quad \forall w_{1}, w_{2} \in H^{1}(\Omega) .
$$

Using Poincaré's inequality we obtain the corresponding coercivity condition

$$
a(w, w) \geq C_{P}\|w\|_{H^{1}(\Omega)}^{2} \quad \forall w \in H_{0}^{1}(\Omega) .
$$

We close this preliminary section, by recalling Young's inequality, a discrete Grönwall lemma, and the GagliardoNirenberg interpolation inequality.

Young's inequality: for any $a, b \geq 0$ any $\delta>0$, and $s_{1}, s_{2}>1$

$$
a b \leq \delta a^{s_{1}}+C(\delta) b^{s_{2}}, \quad \text { with }\left(1 / s_{1}\right)+\left(1 / s_{2}\right)=1 .
$$

Discrete Grönwall lemma: if $a^{n}+b^{n} \leq\left(1+C \tau_{n}\right) a^{n-1}+f^{n}$ and $\tau_{n}$ satisfies the inequality $\max _{n} C \tau_{n}<1$ then

$$
a^{N}+\sum_{n=1}^{N} \mathrm{e}^{C\left(t^{N}-t^{n}\right)} b^{i} \leq(1+T \mathcal{O}(\tau))\left(\mathrm{e}^{C t^{N}} a^{0}+\sum_{n=1}^{N} \mathrm{e}^{C\left(t^{N}-t^{n}\right)} f^{n}\right)
$$

where $\tau=\max _{n} \tau_{n}, \tau_{n}=t^{n}-t^{n-1}$, and $t^{n}=\sum_{i=1}^{n} \tau_{n}$.

Gagliardo-Nirenberg inequality: let $1 \leq q \leq r<\infty$. Then, for $s=1-(q / r)$,

$$
\|u\|_{L^{r}(\Omega)} \leq C\|u\|_{L^{q}(\Omega)}^{1-s}\|u\|_{H^{1}(\Omega)}^{s}, \quad \forall u \in H^{1}(\Omega) .
$$

In particular, we will be using the following interpolation inequality,

$$
\|u\|_{L^{4}(\Omega)}^{2} \leq C\|u\|_{L^{2}(\Omega)}\|u\|_{H^{1}(\Omega)} .
$$

\subsection{Weak formulations}

The following weak formulation of (1.1)-(1.2) will be used subsequently. Suppose that $f_{1}, f_{2} \in$ $L^{2}\left[0, T ; H^{-1}(\Omega)\right]$, and $u_{0}, v_{0} \in L^{2}(\Omega)$. Then, for all $w \in H_{0}^{1}(\Omega)$ and for a.e. $t \in(0, T]$, we seek $u, v \in W(0, T)$ such that

$$
\begin{aligned}
& \left\{\begin{array}{l}
\left\langle u_{t}, w\right\rangle+a(u, w)+\left\langle u^{3}-u, w\right\rangle=\left\langle f_{1}-v, w\right\rangle \\
(u(0), w)=\left(u_{0}, w\right),
\end{array}\right. \\
& \left\{\begin{array}{l}
\left\langle v_{t}, w\right\rangle+\delta a(v, w)=\epsilon\left(u-\alpha_{1} v, w\right)+\left\langle f_{2}, w\right\rangle \\
(v(0), w)=\left(v_{0}, w\right) .
\end{array}\right.
\end{aligned}
$$

An equivalent weak formulation suitable for discontinuous time-stepping schemes considered here is to seek $u, v \in W(0, T)$, satisfying,

$$
\begin{gathered}
(u(T), w(T))+\int_{0}^{T}\left(-\left\langle u, w_{t}\right\rangle+a(u, w)+\left(u^{3}-u, w\right)\right) \mathrm{d} t=\left(u_{0}, w(0)\right)+\int_{0}^{T}\left\langle f_{1}-v, w\right\rangle \mathrm{d} t \\
(v(T), w(T))+\int_{0}^{T}\left(-\left\langle v, w_{t}\right\rangle+\delta a(v, w)\right) \mathrm{d} t=\left(v_{0}, w(0)\right)+\int_{0}^{T}\left(\epsilon\left(u-\alpha_{1} v, w\right)+\left\langle f_{2}, w\right\rangle\right) \mathrm{d} t
\end{gathered}
$$

for all $w \in W(0, T)$. Below, we state the basic solvability result of the weak problem, For rigorous proofs we refer the reader to $[28,37,38,42]$. 
Theorem 2.1. Let $f_{1}, f_{2} \in L^{2}\left[0, T ; H^{-1}(\Omega)\right], u_{0}, v_{0} \in L^{2}(\Omega)$ and $\epsilon, \delta, \alpha_{1}$ positive constants. Then, there exists a unique solution pair $(u, v) \in W(0, T) \equiv L^{2}\left[0, T ; H_{0}^{1}(\Omega)\right] \cap H^{1}\left[0, T ; H^{-1}(\Omega)\right]$ which satisfies the following energy estimate

$$
\|u\|_{W(0, T)}+\|v\|_{W(0, T)} \leq C\left(\left\|f_{1}\right\|_{L^{2}\left[0, T ; H^{-1}(\Omega)\right]}+\left\|u_{0}\right\|_{L^{2}(\Omega)}+\left\|f_{2}\right\|_{L^{2}\left[0, T ; H^{-1}(\Omega)\right]}+\left\|v_{0}\right\|_{L^{2}(\Omega)}\right),
$$

where $C$ depends on $\Omega$, and the parameters $\epsilon, \delta, \alpha_{1}$ and $T$.

Remark 2.2. Here, the size of the norms involved is not necessary small, since for many physical choices of the parameters, standard regularity theory results imply that $C$ may depend upon quantities of the form $(1 / \epsilon),\left(1 / \alpha_{1}\right)$ etc.

\section{The FUlly-DiscRETE SYSTEM}

\subsection{The discontinuous time-stepping approximations}

We discretize the FHN system in time by using a discontinuous time-stepping Galerkin approach, combined with standard conforming finite element subspaces. Approximations will be constructed on a partition $0=t^{0}<$ $t^{1}<\ldots<t^{N}=T$ of $[0, T]$. On each interval of the form $\left(t^{n-1}, t^{n}\right]$ of length $\tau_{n}=t^{n}-t^{n-1}$, a subspace $U_{h}^{n}$ of $H_{0}^{1}(\Omega)$ is specified for all $n=1, \ldots, N$ and it is assumed that each $U_{h}^{n}$ satisfies the classical approximation theory results (see e.g. $[8,23]$ ), on regular triangulations. In particular, we assume that there exists an integer $\ell \geq 1$ and a constant $c>0$ (independent of the mesh-size parameter $h$ ) such that if $w \in H^{l+1}(\Omega) \cap H_{0}^{1}(\Omega)$,

$$
\inf _{w_{h} \in U_{h}^{n}}\left\|w-w_{h}\right\|_{H^{s}(\Omega)} \leq C h^{l+1-s}\|w\|_{H^{l+1}(\Omega)}, \quad 0 \leq l \leq \ell, \quad s=-1,0,1 .
$$

In addition, if the triangulations are quasi-uniform, then there exists a constant $C>0$ such that $\left\|w_{h}\right\|_{H^{1}(\Omega)} \leq$ $C h^{-1}\left\|w_{h}\right\|_{L^{2}(\Omega)}, \quad \forall w_{h} \in U_{h}^{n}$. We also assume that the partition is quasi-uniform in time, i.e., there exists a constant $0<\theta \leq 1$ such that $\theta \tau \leq \min _{n=1, \ldots, N} \tau_{n}$, where $\tau=\max _{n=1, \ldots, N} \tau_{n}$. We seek approximate solutions which belong to the space

$$
\mathcal{U}_{h}=\left\{w_{h} \in L^{2}\left[0, T ; H_{0}^{1}(\Omega)\right]:\left.w_{h}\right|_{\left(t^{n-1}, t^{n}\right]} \in \mathcal{P}_{k}\left[t^{n-1}, t^{n} ; U_{h}^{n}\right]\right\} .
$$

Here $\mathcal{P}_{k}\left[t^{n-1}, t^{n} ; U_{h}^{n}\right]$ denotes the space of polynomials of degree $k$ or less having values in $U_{h}^{n}$. By convention, the functions of $\mathcal{U}_{h}$ are left continuous with right limits and hence will subsequently write $w_{h}^{n}$ for $w_{h}\left(t^{n}\right)=w_{h}\left(t_{-}^{n}\right)$, and $w_{h+}^{n}$ for $w_{h}\left(t_{+}^{n}\right)$. Note that we have also used the following notational abbreviation, $w_{h} \equiv w_{h, \tau}, \mathcal{U}_{h} \equiv \mathcal{U}_{h, \tau}$ etc. Similar notation will be also used for the error $e_{u}=u-u_{h}, e_{v}=v-v_{h}$ functions. Due to a well known embedding result, the solution $u, v$ are in $C\left[0, T ; L^{2}(\Omega)\right]$ so that the jump in the error at $t^{n}$, denoted by $\left[e_{u}^{n}\right]$, is $\left[e_{u}^{n}\right]=\left[u_{h}^{n}\right]=u_{h+}^{n}-u_{h}^{n}$, and by $\left[e_{v}^{n}\right]=v_{h+}^{n}-v_{h}^{n}$ respectively.

The fully discrete system is defined as follows: we seek $u_{h}, v_{h} \in \mathcal{U}_{h}$ such that for every $w_{h} \in \mathcal{U}_{h}$,

$$
\begin{aligned}
& \left(u_{h}^{n}, w_{h}^{n}\right)+\int_{t^{n-1}}^{t^{n}}\left(-\left\langle u_{h}, w_{h t}\right\rangle+a\left(u_{h}, w_{h}\right)+\left(u_{h}^{3}-u_{h}, w_{h}\right)\right) \mathrm{d} t=\left(u_{h}^{n-1}, w_{h+}^{n-1}\right)+\int_{t^{n-1}}^{t^{n}}\left\langle f_{1}-v_{h}, w_{h}\right\rangle \mathrm{d} t \\
& \left(v_{h}^{n}, w_{h}^{n}\right)+\int_{t^{n-1}}^{t^{n}}\left(-\left\langle v_{h}, w_{h t}\right\rangle+\delta a\left(v_{h}, w_{h}\right)\right) \mathrm{d} t=\left(v_{h}^{n-1}, w_{h+}^{n-1}\right)+\int_{t^{n-1}}^{t^{n}}\left(\epsilon\left(u_{h}-\alpha_{1} v_{h}, w_{h}\right)+\left\langle f_{2}, w_{h}\right\rangle\right) \mathrm{d} t .
\end{aligned}
$$

Here, $f_{1}, f_{2} \in L^{2}\left[0, T ; H^{-1}(\Omega)\right]$ are given data, and $u^{0}, v^{0}$ denote approximations of $u_{0}, v_{0} \in L^{2}(\Omega)$ respectively.

Remark 3.1. The existence and uniqueness of discontinuous Galerkin approximations can be proved easily in case $k=0,1$. For the case $k>1$, the existence and (local) uniqueness can be proved around the continuous (smooth) solution $u, v$ (in an appropriate "parabolic" cube), provided that the semi-linear term satisfies 
suitable continuity and monotonicity assumptions which allow the application of standard fixed point theorems (see, e.g., $[2,15,40])$. Existence of discrete schemes of arbitrary order $k$ under minimal regularity assumptions on the data can be proved analogously via fixed point theorems, while uniqueness follows by standard arguments upon deriving stability estimates (see the subsequent section).

\subsection{Approximation of discrete characteristic functions}

In order to obtain stability and error estimates for the system, a key ingredient is the stability result at the interior time points under minimal regularity assumptions. For the latter, we use the theory of the approximation of the discrete characteristic functions (see e.g. [5]), which was used for a general linear parabolic PDE. The main advantage of this approach is that the proof does not need any additional regularity, apart from the one needed to guarantee the existence of a weak solution, i.e., we do not assume that $u_{t}, v_{t} \in L^{2}\left[0, T ; L^{2}(\Omega)\right]$ which is frequently used in the literature for DG approximations of parabolic PDEs. Our main focus is to treat high order (in time) schemes.

Note that the computation of the error at arbitrary times $t \in\left[t^{n-1}, t^{n}\right)$ can be facilitated by substituting $u_{h}=\chi_{\left[t^{n-1}, t\right)} u_{h}$ (similar for $v_{h}$ ) into the discrete equations. However, this choice is not available since $\chi_{\left[t^{n-1}, t\right)} u_{h}$ is not a member of $\mathcal{U}_{h}$, unless $t$ coincides with a partition point. Therefore, approximations of such functions need to be constructed. This is done in [5] Section 2.3. For completeness we state the main results. The approximations are constructed on the interval $(0, \tau)$, and they are invariant under translations.

We consider polynomials $p \in \mathcal{P}_{k}(0, \tau)$, and we denote the discrete approximation of $\chi_{[0, t)} p$ by the polynomial $\tilde{p} \in \mathcal{P}_{k}(0, \tau)$ with, $\tilde{p}(0)=p(0)$ which satisfies

$$
\int_{0}^{\tau} \tilde{p} q=\int_{0}^{t} p q \quad \forall q \in \mathcal{P}_{k-1}(0, \tau) .
$$

To motivate the above construction we simply observe that for $q=p^{\prime}$ we obtain $\int_{0}^{\tau} p^{\prime} \tilde{p}=\int_{0}^{t} p p^{\prime}=\frac{1}{2}\left(p^{2}(t)-\right.$ $\left.p^{2}(0)\right)$.

It is clear that this construction can be extended to approximations of $\chi_{[0, t)} u$ for $u \in \mathcal{P}_{k}[0, \tau ; U]$ where $U$ is a linear space. Note that if $u \in \mathcal{P}_{k}[0, \tau ; U]$ then it can be written as $u=\sum_{i=0}^{k} p_{i}(t) u_{i}$ where $p_{i} \in \mathcal{P}_{k}[0, \tau]$ and $u_{i} \in U$. The discrete approximation of $\chi_{[0, t)} u$ in $\mathcal{P}_{k}[0, \tau ; U]$ is then defined by $\tilde{u}=\sum_{i=0}^{k} \tilde{p}_{i}(t) u_{i}$ and if $U$ is a semi-inner product space we deduce,

$$
\tilde{u}(0)=u(0), \quad \text { and } \int_{0}^{\tau}(\tilde{u}, w)_{U}=\int_{0}^{t}(u, w)_{U} \quad \forall w \in \mathcal{P}_{k-1}[0, \tau ; U]
$$

It remains to quote the main results from $[5,7]$.

Proposition 3.2. Suppose that $U$ is a (semi) inner product space. Then the mapping $\sum_{i=0}^{k} p_{i}(t) u_{i} \rightarrow$ $\sum_{i=0}^{k} \tilde{p}_{i}(t) u_{i}$ on $\mathcal{P}_{k}[0, \tau ; U]$ is continuous in $\|\cdot\|_{L^{2}[0, \tau ; U]}$. In particular,

$$
\|\tilde{u}\|_{L^{2}[0, \tau ; U]} \leq C_{k}\|u\|_{L^{2}[0, \tau ; U]}, \quad\left\|\tilde{u}-\chi_{[0, t)} u\right\|_{L^{2}[0, \tau ; U]} \leq C_{k}\|u\|_{L^{2}[0, \tau ; U]}
$$

where $C_{k}$ is a constant depending on $k$.

Proof. See [5] Lemma 2.4.

A standard calculation gives an explicit formula of $\tilde{u}=\phi(s) z$, when we choose $u(s)=z \in U$ to be constant (see e.g. [7]).

Lemma 3.3. Fix $t \in[0, \tau]$ and let $\phi \in \mathcal{P}_{k}[0, \tau]$ characterized by

$$
\phi(0)=1, \quad \int_{0}^{\tau} \phi q=\int_{0}^{t} q, \quad q \in \mathcal{P}_{k-1}[0, \tau] .
$$


Then,

$$
\phi(s)=1+(s / \tau) \sum_{i=0}^{k-1} c_{i} \hat{p}(s / \tau), \quad c_{i}=\int_{t / \tau}^{1} \hat{p}_{i}(\eta) \mathrm{d} \eta,
$$

where $\left\{\hat{p}_{i}\right\}_{i=0}^{k-1}$ is an orthonormal basis of $\mathcal{P}_{k-1}[0,1]$ in the (weighted) space $L_{w}^{2}[0,1]$ having inner product

$$
(\hat{p}, \hat{q})=\int_{0}^{1} \eta \hat{p}(\eta) \hat{q}(\eta) \mathrm{d} \eta .
$$

In particular, $\|\phi\|_{L^{\infty}[0, \tau]} \leq C_{k}$, where $C_{k}$ is independent of $t \in[0, \tau]$.

\subsection{The main stability estimate}

Now, we are ready to state the main stability result which will be used subsequently to the derivation of estimates at arbitrary time-points. We emphasize that the time-discretization parameter $\tau$ is chosen independent of $h$ and the dependence of various constants on parameters $\epsilon, \delta, \alpha_{1}$ is quantified.

Proposition 3.4. Suppose that $f_{1}, f_{2} \in L^{2}\left[0, T ; H^{-1}(\Omega)\right], u_{0}, v_{0} \in L^{2}(\Omega)$, and let $u_{h}, v_{h}$ be the approximate solutions computed by using the discontinuous time-stepping scheme. Let $\tau_{n}=t^{n}-t^{n-1}$ and define $\tau=\max _{n} \tau_{n}$. Then, there exists constant $C$ depending on $\Omega, C_{k}$, and $T$ such that,

$$
\begin{gathered}
\left\|u_{h}^{n}\right\|_{L^{2}(\Omega)}^{2}+(1 / \epsilon)\left\|v_{h}^{n}\right\|_{L^{2}(\Omega)}^{2}+\left\|u_{h}\right\|_{L^{2}\left[0, T ; H_{0}^{1}(\Omega)\right]}^{2}+\left\|u_{h}\right\|_{L^{4}\left[0, T ; L^{4}(\Omega)\right]}^{4}+\alpha_{1}\left\|v_{h}\right\|_{L^{2}\left[0, T ; L^{2}(\Omega)\right]}^{2}+(\delta / \epsilon)\left\|v_{h}\right\|_{L^{2}\left[0, T ; H_{0}^{1}(\Omega)\right]}^{2} \\
\leq C\left(\left\|u_{0}\right\|_{L^{2}(\Omega)}^{2}+(1 / \epsilon)\left\|v_{0}\right\|_{L^{2}(\Omega)}^{2}+\left\|f_{1}\right\|_{L^{2}\left[0, T ; H^{-1}(\Omega)\right]}^{2}+(1 / \delta \epsilon)\left\|f_{2}\right\|_{L^{2}\left[0, T ; H^{-1}(\Omega)\right]}^{2}\right) \equiv C_{\mathrm{st}} .
\end{gathered}
$$

Here, $\tau$ is chosen to satisfy $\tau \leq\left(C\left(C_{k}, C_{P}\right) / \alpha_{1}\right)$, where $C\left(C_{k}, C_{P}\right)$ is a constant depending only upon $k$ and $\Omega$ but not on $\epsilon, \delta, \alpha_{1}$. In addition,

$$
\left\|u_{h}\right\|_{L^{\infty}\left[0, T ; L^{2}(\Omega)\right]}^{2} \leq C C_{\mathrm{st}}
$$

where $C$ is an algebraic constant independent of $\epsilon, \delta, \alpha_{1}$.

Proof.

Step 1. Preliminary estimates.

Setting $w_{h}=u_{h}, w_{h}=v_{h}$ in (3.1)-(3.2) respectively and using the Poincaré inequality we obtain:

$$
\begin{gathered}
(1 / 2)\left\|u_{h}^{n}\right\|_{L^{2}(\Omega)}^{2}+\int_{t^{n-1}}^{t^{n}}\left(C_{P}\left\|u_{h}\right\|_{H^{1}(\Omega)}^{2}+\left\|u_{h}\right\|_{L^{4}(\Omega)}^{4}\right) \mathrm{d} t+(1 / 2)\left\|\left[u_{h}^{n-1}\right]\right\|_{L^{2}(\Omega)}^{2}-(1 / 2)\left\|u_{h}^{n-1}\right\|_{L^{2}(\Omega)}^{2} \\
\leq \int_{t^{n-1}}^{t^{n}}\left\|u_{h}\right\|_{L^{2}(\Omega)}^{2} \mathrm{~d} t+\int_{t^{n-1}}^{t^{n}}\left\langle f_{1}-v_{h}, u_{h}\right\rangle \mathrm{d} t,
\end{gathered}
$$

and

$$
\begin{aligned}
& (1 / 2)\left\|v_{h}^{n}\right\|_{L^{2}(\Omega)}^{2}+\int_{t^{n-1}}^{t^{n}} C_{P} \delta\left\|v_{h}\right\|_{H^{1}(\Omega)}^{2} \mathrm{~d} t+(1 / 2)\left\|\left[v_{h}^{n-1}\right]\right\|_{L^{2}(\Omega)}^{2}-(1 / 2)\left\|v_{h}^{n-1}\right\|_{L^{2}(\Omega)}^{2} \\
& \leq \int_{t^{n-1}}^{t^{n}} \epsilon\left(u_{h}-\alpha_{1} v_{h}, v_{h}\right) \mathrm{d} t+\int_{t^{n-1}}^{t^{n}}\left\langle f_{2}, v_{h}\right\rangle \mathrm{d} t .
\end{aligned}
$$

Rearranging terms in inequalities (3.3) and (3.4) and using Young's inequality, we obtain respectively

$$
\begin{aligned}
(1 / 2)\left\|u_{h}^{n}\right\|_{L^{2}(\Omega)}^{2}-(1 / 2)\left\|u_{h}^{n-1}\right\|_{L^{2}(\Omega)}^{2} & +\int_{t^{n-1}}^{t^{n}}\left(\left(C_{P} / 2\right)\left\|u_{h}\right\|_{H^{1}(\Omega)}^{2}+\left\|u_{h}\right\|_{L^{4}(\Omega)}^{4}\right) \mathrm{d} t+(1 / 2)\left\|\left[u_{h}^{n-1}\right]\right\|_{L^{2}(\Omega)}^{2} \\
\leq & -\int_{t^{n-1}}^{t^{n}}\left(v_{h}, u_{h}\right) \mathrm{d} t+\int_{t^{n-1}}^{t^{n}}\left\|u_{h}\right\|_{L^{2}(\Omega)}^{2} \mathrm{~d} t+\int_{t^{n-1}}^{t^{n}}\left(1 / C_{P}\right)\left\|f_{1}\right\|_{H^{-1}(\Omega)}^{2} \mathrm{~d} t
\end{aligned}
$$


and

$$
\begin{array}{r}
(1 / 2)\left\|v_{h}^{n}\right\|_{L^{2}(\Omega)}^{2}-(1 / 2)\left\|v_{h}^{n-1}\right\|_{L^{2}(\Omega)}^{2}+\int_{t^{n-1}}^{t^{n}}\left(\left(C_{P} \delta / 2\right)\left\|v_{h}\right\|_{H^{1}(\Omega)}^{2}+\epsilon \alpha_{1}\left\|v_{h}\right\|_{L^{2}(\Omega)}^{2}\right) \mathrm{d} t+(1 / 2)\left\|\left[v_{h}^{n-1}\right]\right\|_{L^{2}(\Omega)}^{2} \\
\leq \int_{t^{n-1}}^{t^{n}} \epsilon\left(u_{h}, v_{h}\right) \mathrm{d} t+\left(1 / 2 C_{P} \delta\right) \int_{t^{n-1}}^{t^{n}}\left\|f_{2}\right\|_{H^{-1}(\Omega)}^{2} \mathrm{~d} t .
\end{array}
$$

Multiplying equation (3.5) by $\epsilon$ and adding the resulting equation to (3.6), we arrive at the following preliminary estimate:

$$
\begin{gathered}
\epsilon\left\{(1 / 2)\left\|u_{h}^{n}\right\|_{L^{2}(\Omega)}^{2}-(1 / 2)\left\|u_{h}^{n-1}\right\|_{L^{2}(\Omega)}^{2}+\int_{t^{n-1}}^{t^{n}}\left(\left(C_{P} / 2\right)\left\|u_{h}\right\|_{H^{1}(\Omega)}^{2}+\left\|u_{h}\right\|_{L^{4}(\Omega)}^{4}\right) \mathrm{d} t+(1 / 2)\left\|\left[u_{h}^{n-1}\right]\right\|_{L^{2}(\Omega)}^{2}\right\} \\
+(1 / 2)\left\|v_{h}^{n}\right\|_{L^{2}(\Omega)}^{2}-(1 / 2)\left\|v_{h}^{n-1}\right\|_{L^{2}(\Omega)}^{2}+\int_{t^{n-1}}^{t^{n}}\left(C_{P} \delta / 2\right)\left\|v_{h}\right\|_{H^{1}(\Omega)}^{2} \mathrm{~d} t+\int_{t^{n-1}}^{t^{n}} \epsilon \alpha_{1}\left\|v_{h}\right\|_{L^{2}(\Omega)}^{2} \mathrm{~d} t+(1 / 2)\left\|\left[v_{h}^{n-1}\right]\right\|_{L^{2}(\Omega)}^{2} \\
\leq \int_{t^{n-1}}^{t^{n}} \epsilon\left\|u_{h}\right\|_{L^{2}(\Omega)}^{2} \mathrm{~d} t+\int_{t^{n-1}}^{t^{n}} C_{P} \epsilon\left\|f_{1}\right\|_{H^{-1}(\Omega)}^{2} \mathrm{~d} t+\left(1 / 2 C_{P} \delta\right) \int_{t^{n-1}}^{t^{n}}\left\|f_{2}\right\|_{H^{-1}(\Omega)}^{2} \mathrm{~d} t . \quad(3.7)
\end{gathered}
$$

Step 2. Estimates based on the discrete characteristic function.

Recall that for $k=0,1$ one may hide the first term on the left to obtain an initial estimate for $u_{h}, v_{h}$ upon summation from 0 to $N$ by using standard techniques. For the high order schemes, we employ properties of the discrete characteristic and its approximation by following the technique of $[7,41]$. For fixed $t \in\left[t^{n-1}, t^{n}\right)$ and $z_{h} \in U_{h}^{n}$ we substitute $w_{h}(s)=z_{h} \phi(s)$ into (3.1), where $\phi(s) \in \mathcal{P}_{k}\left[t^{n-1}, t^{n}\right]$ is constructed similar to Lemma 3.3, i.e.,

$$
\phi\left(t^{n-1}\right)=1, \quad \int_{t^{n-1}}^{t^{n}} \phi q=\int_{t^{n-1}}^{t} q, \quad q \in \mathcal{P}_{k-1}\left[t^{n-1}, t^{n}\right] .
$$

Recall that Lemma 3.3 asserts that $\|\phi\|_{L^{\infty}} \leq C_{k}$, with $C_{k}$ independent of $t$. Now, it is easy to see that with this particular choice of $w_{h}$,

$$
\begin{aligned}
\int_{t^{n-1}}^{t^{n}}\left(u_{h t}, w_{h}\right) \mathrm{d} s+\left(u_{h+}^{n-1}-u_{h}^{n-1}, w_{h+}^{n-1}\right) & =\int_{t^{n-1}}^{t}\left(u_{h t}, z_{h}\right) \mathrm{d} s+\left(u_{h+}^{n-1}-u_{h}^{n-1}, \phi\left(t^{n-1}\right) z_{h}\right) \\
& =\left(u_{h}(t)-u_{h}^{n-1}, z_{h}\right) .
\end{aligned}
$$

Hence integrating by parts (in time) equation (3.1) and using the above computation, we obtain

$$
\begin{aligned}
\left(u_{h}(t)-u_{h}^{n-1}, z_{h}\right)= & -\int_{t^{n-1}}^{t^{n}}\left(a\left(u_{h}, z_{h}\right)+\left(u_{h}^{3}-u_{h}, z_{h}\right)\right) \phi \mathrm{d} s+\int_{t^{n-1}}^{t^{n}}\left\langle f_{1}-v_{h}, z_{h}\right\rangle \phi \mathrm{d} s \\
\leq & C_{k}\left\{\int_{t^{n-1}}^{t^{n}}\left(\left\|\nabla u_{h}\right\|_{L^{2}(\Omega)}\left\|\nabla z_{h}\right\|_{L^{2}(\Omega)}+\left\|u_{h}\right\|_{L^{2}(\Omega)}\left\|z_{h}\right\|_{L^{2}(\Omega)}\right) \mathrm{d} s\right. \\
& \left.+\int_{t^{n-1}}^{t^{n}}\left(\left\|u_{h}^{3}\right\|_{L^{4 / 3}(\Omega)}\left\|z_{h}\right\|_{L^{4}(\Omega)}+\left\|v_{h}\right\|_{L^{2}(\Omega)}\left\|z_{h}\right\|_{L^{2}(\Omega)}\right) \mathrm{d} s+\int_{t^{n-1}}^{t^{n}}\left\|f_{1}\right\|_{H^{-1}(\Omega)}\left\|z_{h}\right\|_{H^{1}(\Omega)} \mathrm{d} s\right\},
\end{aligned}
$$

where we have used Lemma 3.3 to bound $\|\phi\|_{L^{\infty}} \leq C_{k}$ with $C_{k}$ denoting a constant depending only on $k, \Omega$. Note also that $z_{h} \in U_{h}^{n}$ (independent of $s$ ), hence the above inequality leads to

$$
\begin{aligned}
\left(u_{h}(t)-u_{h}^{n-1}, z_{h}\right) \leq & C_{k}\left[\int_{t^{n-1}}^{t^{n}}\left(\left\|u_{h}\right\|_{H^{1}(\Omega)}+\left\|v_{h}\right\|_{L^{2}(\Omega)}+\left\|f_{1}\right\|_{H^{-1}(\Omega)}\right) \mathrm{d} s\right]\left\|z_{h}\right\|_{H^{1}(\Omega)} \\
& +C_{k}\left[\int_{t^{n-1}}^{t^{n}}\left\|u_{h}\right\|_{L^{4}(\Omega)}^{3} \mathrm{~d} s\right]\left\|z_{h}\right\|_{L^{4}(\Omega)} .
\end{aligned}
$$


Here we have used the fact $\left\|u_{h}^{3}\right\|_{L^{4 / 3}(\Omega)} \leq C\left\|u_{h}\right\|_{L^{4}(\Omega)}^{3}$. Setting $z_{h}=u_{h}(t)$ (for the previously fixed $t \in\left[t^{n-1}, t^{n}\right)$ ), using Hölder's inequality, and integrating in time the resulting inequality, we obtain,

$$
\begin{aligned}
& (1 / 2) \int_{t^{n-1}}^{t^{n}}\left\|u_{h}(t)\right\|_{L^{2}(\Omega)}^{2} \mathrm{~d} t \leq\left(\tau_{n} / 2\right)\left\|u_{h}^{n-1}\right\|_{L^{2}(\Omega)}^{2} \\
& \quad+C_{k} \tau_{n}^{1 / 2}\left(\left\|u_{h}\right\|_{L^{2}\left[t^{n-1}, t^{n} ; H^{1}(\Omega)\right]}+\left\|v_{h}\right\|_{L^{2}\left[t^{n-1}, t^{n} ; L^{2}(\Omega)\right]}+\left\|f_{1}\right\|_{L^{2}\left[t^{n-1}, t^{n} ; H^{-1}(\Omega)\right]}\right) \\
& \quad \times \int_{t^{n-1}}^{t^{n}}\left\|u_{h}(t)\right\|_{H^{1}(\Omega)} \mathrm{d} t+C_{k} \tau_{n}^{1 / 4}\left\|u_{h}\right\|_{L^{4}\left[t^{n-1}, t^{n} ; L^{4}(\Omega)\right]}^{3} \int_{t^{n-1}}^{t^{n}}\left\|u_{h}(t)\right\|_{L^{4}(\Omega)} \mathrm{d} t .
\end{aligned}
$$

Using Hölder's inequality to bound $\int_{t^{n-1}}^{t^{n}}\left\|u_{h}\right\|_{L^{4}(\Omega)} \mathrm{d} t \leq \tau_{n}^{3 / 4}\left\|u_{h}\right\|_{L^{4}\left[t^{n-1}, t^{n} ; L^{4}(\Omega)\right]}, \quad \int_{t^{n-1}}^{t^{n}}\left\|u_{h}\right\|_{H^{1}(\Omega)} \mathrm{d} t \leq$ $\tau_{n}^{1 / 2}\left\|u_{h}\right\|_{L^{2}\left[t^{n-1}, t^{n} ; H^{1}(\Omega)\right]}$, and Young's inequalities we deduce (with different $C_{k}$ ),

$$
\begin{aligned}
(1 / 2) & \int_{t^{n-1}}^{t^{n}}\left\|u_{h}(t)\right\|_{L^{2}(\Omega)}^{2} \mathrm{~d} t \leq\left(\tau_{n} / 2\right)\left\|u_{h}^{n-1}\right\|_{L^{2}(\Omega)}^{2} \\
& +C_{k} \tau_{n}\left(\left\|u_{h}\right\|_{L^{2}\left[t^{n-1}, t^{n} ; H^{1}(\Omega)\right]}^{2}+\left\|v_{h}\right\|_{L^{2}\left[t^{n-1}, t^{n} ; L^{2}(\Omega)\right]}^{2}+\left\|f_{1}\right\|_{L^{2}\left[t^{n-1}, t^{n} ; H^{-1}(\Omega)\right]}^{2}\right) \\
& +C_{k} \tau_{n}\left\|u_{h}\right\|_{L^{4}\left[t^{n-1}, t^{n} ; L^{4}(\Omega)\right]}^{4} .
\end{aligned}
$$

\section{Step 3. Combining estimates.}

The final step is to substitute the value of $\left\|u_{h}\right\|_{L^{2}\left[t^{n-1}, t^{n} ; L^{2}(\Omega)\right]}^{2}$ in (3.7) by the given bound of (3.9). Therefore,

$$
\begin{aligned}
& \epsilon\left\|u_{h}^{n}\right\|_{L^{2}(\Omega)}^{2}+ \epsilon\left(C_{P}-C_{k} \tau_{n}\right) \int_{t^{n-1}}^{t^{n}}\left\|u_{h}\right\|_{H^{1}(\Omega)}^{2} \mathrm{~d} t+2 \epsilon\left(1-C_{k} \tau_{n}\right) \int_{t^{n-1}}^{t^{n}}\left\|u_{h}\right\|_{L^{4}(\Omega)}^{4}+\left\|v_{h}^{n}\right\|_{L^{2}(\Omega)}^{2}+\epsilon\left(a_{1}-C_{k} \tau_{n}\right) \\
& \times \int_{t^{n-1}}^{t^{n}}\left\|v_{h}\right\|_{L^{2}(\Omega)}^{2} \mathrm{~d} t+C_{P} \delta \int_{t^{n-1}}^{t^{n}}\left\|v_{h}\right\|_{H^{1}(\Omega)}^{2} \mathrm{~d} t \leq \epsilon\left(1+C_{k} \tau_{n}\right)\left\|u_{h}^{n-1}\right\|_{L^{2}(\Omega)}^{2}+\left\|v_{h}^{n-1}\right\|_{L^{2}(\Omega)}^{2} \\
&+C\left(C_{k}, C_{p}\right)\left[\int_{t^{n-1}}^{t^{n}}\left(\left(\epsilon+\epsilon \tau_{n}\right)\left\|f_{1}\right\|_{H^{-1}(\Omega)}^{2}+(1 / \delta)\left\|f_{2}\right\|_{H^{-1}(\Omega)}^{2}\right) \mathrm{d} t\right] .
\end{aligned}
$$

Selecting $\tau$ such that $\tau_{n} \leq\left(1 / C_{k} \alpha_{1}\right)$, and $\tau_{n} \leq\left(C_{P} / C_{k}\right)$ and using standard discrete Grönwall lemma arguments, we obtain the estimates at partition points and at the energy norms, upon dividing by $\epsilon$. Returning back to (3.9) using the inverse estimate $\left\|u_{h}(t)\right\|_{L^{2}(\Omega)}^{2} \leq\left(C_{k} / \tau_{n}\right) \int_{t^{n-1}}^{t^{n}}\left\|u_{h}\right\|_{L^{2}(\Omega)}^{2}$ and the previously developed estimates we obtain the estimate,

$$
\begin{aligned}
\left\|u_{h}\right\|_{L^{\infty}\left[t^{n-1}, t^{n} ; L^{2}(\Omega)\right]}^{2} \leq & C_{k}\left(\left\|u_{h}^{n}\right\|_{L^{2}(\Omega)}^{2}+\left\|u_{h}\right\|_{L^{2}\left[t^{n-1}, t^{n} ; H^{1}(\Omega)\right]}^{2}+\left\|v_{h}\right\|_{L^{2}\left[t^{n-1}, t^{n} ; L^{2}(\Omega)\right]}^{2}\right. \\
& \left.+\left\|f_{1}\right\|_{L^{2}\left[t^{n-1}, t^{n} ; H^{-1}(\Omega)\right]}^{2}+\left\|u_{h}\right\|_{L^{4}\left[t^{n-1}, t^{n} ; L^{4}(\Omega)\right]}^{4}\right) .
\end{aligned}
$$

The estimate now follows by substituting the estimates at the partition points and at energy norms.

Remark 3.5. The above theorem states that the discontinuous Galerkin discretization inherits the stability estimates of the weak formulation under minimal regularity assumptions on the given data.

Remark 3.6. The stability estimate at arbitrary time-points for $v_{h}$ can be obtained identically to [5] Section 2 , since we have already obtained an estimate on $u_{h}, v_{h}$ in $L^{2}\left[0, T ; H^{1}(\Omega)\right]$. 


\subsection{Convergence under minimal regularity assumptions}

Using the approach of [41] we may recover strong convergence in an appropriate norm, and pass the limit through the nonlinear term. Hence, the convergence of the space-time approximations can be shown even under minimal regularity assumptions. First, we quote a general compactness result by Walkington (see [41] Thm. 3.1), for discontinuous time-stepping schemes, that concerns the numerical approximations of solutions $y:[0, T] \rightarrow X$ of general evolution equations of the form

$$
y_{t}+A(y)=f(y), \quad y(0)=y_{0},
$$

where $X$ is a Banach space and each term of the equation takes values in $X^{*}$. Here, both $A(y)=A(t, y)$ and $f(y)=f(t, y)$ may depend upon $t$ and are allowed to be nonlinear. However, in our setting only $f(y)=f(t, y)$ contains nonlinear terms. Suppose that $X \subset H \subset X^{*}$ (with continuous embeddings) form the standard evolution triple, i.e., the pivot space $H$ is a Hilbert space. The numerical schemes approximate the weak form of (3.10), i.e.,

$$
\left\langle y_{t}, w\right\rangle+a(y, w)=\langle f(y), w\rangle, \quad \forall w \in X
$$

where $a: X \times X \rightarrow \mathbb{R}$ is defined by $a(y, w)=(A(y), w)$. Let $U_{h} \subset X$ and quasi-uniform partition $0=t^{0}<$ $t^{1}<\ldots<t^{N}=T$ of $[0, T]$, and set $U_{h}^{n} \equiv U_{h}$, for $n=1, \ldots, N$. Then, the DG scheme constructs a function in $\mathcal{P}_{k}\left[t^{n-1}, t^{n} ; U_{h}\right]$ on each $\left(t^{n-1}, t^{n}\right)$, which satisfies for $n=1, \ldots, N$ and for all $w_{h} \in \mathcal{P}_{k}\left[t^{n-1}, t^{n} ; U_{h}\right]$,

$$
\int_{t^{n-1}}^{t^{n}}\left(\left(y_{h t}, w_{h}\right)+a\left(y_{h}, w_{h}\right)\right)+\left(y_{h+}^{n-1}-y_{h}^{n-1}, w_{h+}^{n-1}\right)=\int_{t^{n-1}}^{t^{n}}\left(f\left(y_{h}\right), w_{h}\right)
$$

Here, $y_{h}^{0}$ is a given approximation of $y_{0}$. Set $F(y) \equiv f(y)-A(y)$. Then the following theorem [41] Theorem 3.1, establishes the compactness property of the discrete approximation.

Theorem 3.7. Let $H$ be a Hilbert space, $X$ be a Banach space and $X \subset H \subset X^{*}$ be dense and compact embeddings. Fix an integer $k \geq 0$ and let $1 \leq p, q<\infty$. Let $h>0$ be the mesh parameter, and let $\left\{t^{i}\right\}_{i=0}^{N}$ denote a quasi-uniform partition of $[0, T]$. Assume that:

1. for each $h>0, y_{h} \in\left\{y_{h} \in L^{p}[0, T ; U]\left|y_{h}\right|_{\left(t^{n-1}, t^{n}\right)} \in \mathcal{P}_{k}\left[t^{n-1}, t^{n} ; U_{h}\right]\right\}$ and on each interval, satisfies

$$
\int_{t^{n-1}}^{t^{n}}\left(y_{h t}, w_{h}\right)+\left(y_{h+}^{n-1}-y_{h}^{n-1}, w_{h+}^{n-1}\right)=\int_{t^{n-1}}^{t^{n}}\left(F\left(y_{h}\right), w_{h}\right)
$$

for every $w_{h} \in \mathcal{P}_{k}\left[t^{n-1}, t^{n} ; U_{h}\right]$;

2. $\left\{y_{h}\right\}_{h>0}$ is bounded in $L^{p}[0, T ; U]$ and $\left\{\left\|F\left(y_{h}\right)\right\|_{L^{q}\left[0, T ; U^{*}\right]}\right\}_{h>0}$ is also bounded.

Then,

1. if $p>1$ then $\left\{y_{h}\right\}_{h>0}$ is compact in $L^{r}[0, T ; H]$ for $1 \leq r<2 p$;

2. if $1 \leq(1 / p)+(1 / q)<2$, and $\sum_{i=1}^{N}\left\|\left[y_{h}\right]\right\|_{H}^{2}<C$ is bounded independent of $h$, then $\left\{y_{h}\right\}_{h>0}$ is compact in $L^{r}[0, T ; H]$ for $1 \leq r<2 /((1 / p)+(1 / q)-1)$.

Proof. See [41] Theorem 3.1.

We will utilize the above result to obtain strong convergence of the discrete (FHN) system to the continuous one. The lack of any meaningful regularity for the discrete time derivative due to the presence of discontinuities, requires special attention since the classical Aubin-Lions compactness argument is not directly applicable. 
Theorem 3.8. Suppose that $f_{1}, f_{2} \in L^{2}\left[0, T ; H^{-1}(\Omega)\right], u_{0}, v_{0} \in L^{2}(\Omega)$, and let $\epsilon, \delta, \alpha_{1}$ be given parameters. Let $\left\{t^{i}\right\}_{i=0}^{N}$ denote a quasi-uniform partition of $[0, T]$, and $U_{h}^{n} \equiv U_{h}$ for $n=1, \ldots, N$. Suppose that the assumptions of Proposition 3.4 hold, and let $\tau, h \rightarrow 0$. Then,

$$
\begin{aligned}
& u_{h} \rightarrow u \text { weakly in } L^{2}\left[0, T ; H_{0}^{1}(\Omega)\right], \quad u_{h} \rightarrow u \text { weakly-* in } L^{\infty}\left[0, T ; L^{2}(\Omega)\right], \\
& v_{h} \rightarrow v \text { weakly in } L^{2}\left[0, T ; H_{0}^{1}(\Omega)\right], \quad v_{h} \rightarrow v \text { weakly-* in } L^{\infty}\left[0, T ; L^{2}(\Omega)\right],
\end{aligned}
$$

and

$$
u_{h} \rightarrow u \quad \text { stronly in } L^{2}\left[0, T ; L^{2}(\Omega)\right], \quad v_{h} \rightarrow v \quad \text { strongly in } L^{2}\left[0, T ; L^{2}(\Omega)\right] .
$$

In addition $(u, v)$ is a weak solution of the (FHN) system.

Proof. We follow the same line of arguments with [41] Section 6. The stability estimates of Proposition 3.4 clearly imply that (passing to a subsequence if necessary) there exist $u, v$ such that $u_{h} \rightarrow u$ and $v_{h} \rightarrow v$ weakly in $L^{2}\left[0, T ; H_{0}^{1}(\Omega)\right]$ and weakly-* in $L^{\infty}\left[0, T ; L^{2}(\Omega)\right]$. It remains to obtain strong convergence in $L^{2}\left[0, T ; L^{2}(\Omega)\right]$. For this purpose, fix $X=H_{0}^{1}(\Omega), H=L^{2}(\Omega)$, and $F(u)=\Delta u-u^{3}+u-v+f_{1}$. It is easy to show that $F\left(u_{h}\right) \in L^{4 / 3}\left[0, T ; H^{-1}(\Omega)\right]$. Indeed, $u_{h} \in L^{2}\left[0, T ; H_{0}^{1}(\Omega)\right] \cap L^{4}\left[0, T ; L^{4}(\Omega)\right]$, and $u_{h} \in L^{\infty}\left[0, T ; L^{2}(\Omega)\right]$ clearly imply that $u_{h}^{3} \in L^{4 / 3}\left[0, T ; H^{-1}(\Omega)\right]$ by using standard interpolation theorems. The remaining terms can be handled easily. Therefore, using the Theorem 3.7, we obtain the desired strong convergence in $L^{2}\left[0, T ; L^{2}(\Omega)\right]$. The strong convergence on $v$ follows similarly and more easily. Suppose now that we choose $w_{h} \in C\left[0, T ; U_{h}\right] \cap \mathcal{U}_{h}$, with $w_{h}(T)=0$. Then, summing equations (3.1) from $n=1$ to $n=N$, we deduce that

$\left(u_{h}(T), w_{h}(T)\right)+\int_{0}^{T}\left(-\left\langle u_{h}, w_{h t}\right\rangle+a\left(u_{h}, w_{h}\right)+\left\langle u_{h}^{3}-u_{h}, w_{h}\right\rangle\right) \mathrm{d} t=\int_{0}^{T}\left(\left\langle f_{1}, w_{h}\right\rangle-\lambda\left\langle v_{h}, w_{h}\right\rangle_{\Gamma}\right) \mathrm{d} t+\left(u^{0}, w_{h}(0)\right)$.

Note that we may pass the limit through the linear terms due to the stability estimates on $u_{h}, v_{h}$ and the fact that $w_{h} \in C\left[0, T ; U_{h}\right] \cap \mathcal{U}_{h}$. The semilinear term can be treated easily by the strong convergence on $L^{2}\left[0, T ; L^{2}(\Omega)\right]$, by noting that $\int_{0}^{T}\left|\left\langle u_{h}^{3}-u^{3}, w_{h}\right\rangle\right| \mathrm{d} t \leq C_{u, u_{h}}\left\|u-u_{h}\right\|_{L^{2}\left[0, T ; L^{2}(\Omega)\right]}^{2}$, where $C\left(u, u_{h}\right)$ denotes a constant that depends upon $\|\cdot\|_{L^{\infty}\left[0, T ; L^{2}(\Omega)\right]},\|\cdot\|_{L^{2}\left[0, T ; H^{1}(\Omega)\right]}$, the stability constants of Proposition 3.4 and it is independent of $h, \tau$. A standard density argument, now completes the proof. The equation of $v_{h}$ can be treated similarly and more easily.

Remark 3.9. The above methodology can be clearly applied in other choices of FigzHugh-Nagumo type of models. Note that strong convergence can be also obtained in $L^{r}\left[0, T ; L^{2}(\Omega)\right]$ for $r \in[1,4)$. In Section 5 , we present some numerical experiments that demonstrate convergence of the DG approximations under minimal regularity assumptions $u_{0}, v_{0} \in L^{2}(\Omega)$, as predicted in Theorem 3.8 .

\section{ERror estimates}

So far we have shown the basic stability estimates in the natural energy norm, as well as at the arbitrary time-points. Recall that, due to the presence of time discontinuities, the discrete time derivative does not possess any meaningful regularity. Hence, for the error estimates, we will also rely on various techniques developed in the previous section which circumvent the lack of regularity. The main strategy is to relate the error $e_{u}=u-u_{h}$ and $e_{v}=v-v_{h}$ to a best approximation error viaan auxiliary uncoupled pair of parabolic PDEs. These auxiliary PDEs play the role of a "global DG projection". 


\subsection{The auxiliary parabolic system}

First, we construct a suitable global "parabolic projection". For example, given data $F_{1}, F_{2}$ satisfying the regularity assumptions of Sections 2 and 3 , we denote by $u_{\mathrm{ph}}, v_{\mathrm{ph}}$ the DG approximations of the following decoupled systems: for all $w_{h} \in \mathcal{U}_{h}$, for $n=1, \ldots, N$, we seek $u_{\mathrm{ph}}, v_{\mathrm{ph}} \in \mathcal{U}_{h}$,

$$
\begin{aligned}
& \left(u_{\mathrm{ph}}^{n}, w_{h}^{n}\right)+\int_{t^{n-1}}^{t^{n}}\left(-\left\langle u_{\mathrm{ph}}, w_{h t}\right\rangle+a\left(u_{\mathrm{ph}}, w_{h}\right)\right) \mathrm{d} t=\left(u_{\mathrm{ph}}^{n-1}, w_{h+}^{n-1}\right)+\int_{t^{n-1}}^{t^{n}}\left\langle F_{1}, w_{h}\right\rangle \mathrm{d} t, \\
& \left(v_{\mathrm{ph}}^{n}, w_{h}^{n}\right)+\int_{t^{n-1}}^{t^{n}}\left(-\left\langle v_{\mathrm{ph}}, w_{h t}\right\rangle+\delta a\left(v_{h}, w_{h}\right)\right) \mathrm{d} t=\left(v_{\mathrm{ph}}^{n-1}, w_{h+}^{n-1}\right)+\int_{t^{n-1}}^{t^{n}}\left\langle F_{2}, w_{h}\right\rangle \mathrm{d} t .
\end{aligned}
$$

Here, $u_{p}^{0} \equiv u_{0}, v_{p}^{0} \equiv v_{0}$. The given data $F_{1}, F_{2} \in L^{2}\left[0, T ; H^{-1}(\Omega)\right]$, are defined as follows:

$$
\begin{aligned}
\left\langle F_{1}, w_{h}\right\rangle & \equiv\left\langle u_{t}-\Delta u, w_{h}\right\rangle \quad \forall w_{h} \in \mathcal{U}_{h} \\
\left\langle F_{2}, w_{h}\right\rangle & \equiv\left\langle v_{t}-\delta \Delta v, w_{h}\right\rangle \quad \forall w_{h} \in \mathcal{U}_{h} .
\end{aligned}
$$

Note that the above system consists of two uncoupled problems, and hence working similarly to [5,7], we obtain the following optimal convergence rates for $u-u_{\mathrm{ph}}, v-v_{\mathrm{ph}}$ for suitably smooth solutions $u, v$. These estimates allows the choice of "coarse" time-steps, while the coercivity constant $\delta>0$ is carefully tracked.

Theorem 4.1. Suppose that the time-steps are quasi-uniform, i.e., there exists $0<\theta \leq 1$ such that $\theta \max _{1 \leq n \leq N} \tau_{n} \leq \min _{1 \leq n \leq N} \tau_{n}$ where $\tau_{n}=t^{n}-t^{n-1}$. Set, $\tau=\max _{n=1, \ldots, N} \tau_{n}$. Then if $u, v \in C\left[0, T ; H^{l+1}(\Omega)\right] \cap$ $H^{k+1}\left[0, T ; H_{0}^{1}(\Omega)\right]$ there exists a constant $C>0$ independent of $h, \tau, \delta$ such that,

$$
\begin{aligned}
\left\|u-u_{\mathrm{ph}}\right\|_{L^{2}\left[0, T ; H^{1}(\Omega)\right]} \leq & C\left(\|u\|_{L^{2}\left[0, T ; H^{l+1}(\Omega)\right]} h^{l}+\left\|u^{(k+1)}\right\|_{L^{2}\left[0, T ; H^{1}(\Omega)\right]} \tau^{k+1}\right. \\
& \left.+\|u\|_{C\left[0, T ; H^{l+1}(\Omega)\right]} \min \left(h^{2} / \tau, h / \sqrt{\tau}\right) h^{l}\right) \\
\left\|v-v_{\mathrm{ph}}\right\|_{L^{2}\left[0, T ; H^{1}(\Omega)\right]} \leq & C\left(\|v\|_{L^{2}\left[0, T ; H^{l+1}(\Omega)\right.} h^{l}+\left\|v^{(k+1)}\right\|_{L^{2}\left[0, T ; H^{1}(\Omega)\right]} \tau^{k+1}\right. \\
& \left.+\|v\|_{C\left[0, T ; H^{l+1}(\Omega)\right]} \min \left(h^{2} /(\tau \delta), h / \sqrt{\tau \delta}\right) h^{l}\right) \\
\left\|u-u_{\mathrm{ph}}\right\|_{L^{\infty}\left[0, T ; L^{2}(\Omega)\right] \leq} & C\left(\left(\|u\|_{L^{2}\left[0, T ; H^{l+1}(\Omega)\right]}+h\|u\|_{L^{\infty}\left[0, T ; H^{l+1}(\Omega)\right]}\right) h^{l}\right. \\
& +\left(\left\|u^{(k+1)}\right\|_{L^{2}\left[0, T ; H^{1}(\Omega)\right]}+\left\|u^{(k+1)}\right\|_{L^{\infty}\left[0, T ; L^{2}(\Omega)\right]}\right) \tau^{k+1} \\
& \left.+\|u\|_{C\left[0, T ; H^{l+1}(\Omega)\right]} \min \left(h^{3 / 2} / \tau, \sqrt{h / \tau}\right) h^{l+1 / 2}\right) \\
\left\|v-v_{\mathrm{ph}}\right\|_{L^{\infty}\left[0, T ; L^{2}(\Omega)\right]} \leq & C\left(\left(\sqrt{\delta}\|v\|_{L^{2}\left[0, T ; H^{l+1}(\Omega)\right.}+h\|v\|_{L^{\infty}\left[0, T ; H^{l+1}(\Omega)\right]}\right) h^{l}\right. \\
& +\left(\sqrt{\delta}\left\|v^{(k+1)}\right\|_{L^{2}\left[0, T ; H^{1}(\Omega)\right]}+\left\|v^{(k+1)}\right\|_{L^{\infty}\left[0, T ; L^{2}(\Omega)\right]}\right) \tau^{k+1} \\
& \left.+\|v\|_{C\left[0, T ; H^{l+1}(\Omega)\right]} \min \left(h^{3 / 2} /(\tau \sqrt{\delta}), \sqrt{h / \tau}\right) h^{l+1 / 2}\right) .
\end{aligned}
$$

If in addition, $F_{1} \in L^{2}\left[0, T ; L^{2}(\Omega)\right], u_{0} \in H_{0}^{1}(\Omega)$, and the domain $\Omega$ is such that classical elliptic regularity is valid then, $\left\|u_{\mathrm{ph}}\right\|_{L^{\infty}\left[0, T ; H^{1}(\Omega)\right]} \leq C_{\mathrm{st}}^{\prime}$, and

$\left\|u-u_{\mathrm{ph}}\right\|_{L^{2}\left[0, T ; L^{2}(\Omega)\right]} \leq C\left(\|u\|_{L^{\infty}\left[0, T ; H^{l+1}(\Omega)\right]} h^{l+1}+\left\|u^{(k+1)}\right\|_{L^{2}\left[0, T ; H^{1}(\Omega)\right]} \tau^{k+1}+\left\|u^{(k+1)}\right\|_{L^{\infty}\left[0, T ; L^{2}(\Omega)\right]} \tau^{k+1}\right)$. 


\section{Proof. (Sketch)}

The proof is essentially identical to that in $[5,7]$ since the system is now decoupled. Starting from the estimate for $v-v_{\mathrm{ph}}$, we split the error as $v-v_{\mathrm{ph}}=\left(v-\mathbb{P}_{h}^{\text {loc }} v\right)+\left(\mathbb{P}_{h}^{\text {loc }} v-v_{\mathrm{ph}}\right)$, where $\mathbb{P}_{h}^{\text {loc }}$ denotes a suitable locally defined projection for discontinuous time-stepping schemes, (see e.g. [5] Def. 2.1 or [40]). Now, observe that the error can be expressed in terms of local $L^{2}$ projections, since [5] Theorem 2.5 asserts that

$$
\begin{aligned}
& \left\|\mathbb{P}_{h}^{\text {loc }} v(t)-v_{\mathrm{ph}}(t)\right\|_{L^{2}(\Omega)}^{2}+\delta \int_{0}^{t^{n}}\left\|\nabla\left(\mathbb{P}_{h}^{\mathrm{loc}} v-v_{\mathrm{ph}}\right)(t)\right\|_{L^{2}(\Omega)}^{2} \mathrm{~d} t+\sum_{i=0}^{n-1}\left\|\left[\left(\mathbb{P}_{h}^{\mathrm{loc}} v-v_{\mathrm{ph}}\right)^{i}\right]\right\|_{L^{2}(\Omega)}^{2} \\
& \leq C_{k}\left(\left\|v_{0}-v_{h}^{0}\right\|_{L^{2}(\Omega)}^{2}+\delta \int_{0}^{t^{n}}\left\|v-\mathbb{P}_{h}^{\mathrm{loc}} v\right\|_{H^{1}(\Omega)}^{2} \mathrm{~d} t\right. \\
& \left.\quad+\sum_{i=0}^{n-1} \min \left(1 /\left(\tau_{i} \delta\right)\left\|P_{i+1}\left(I-P_{i}\right) v\left(t^{i}\right)\right\|_{H^{-1}(\Omega)}^{2},\left\|\left(I-P_{i}\right) v\left(t^{i}\right)\right\|_{L^{2}(\Omega)}^{2}\right)\right)
\end{aligned}
$$

where $P_{i}: L^{2}(\Omega) \rightarrow U_{h}^{i}$ denotes the standard $L^{2}$ projection. Now it remains to combine approximation theory results (see e.g. [6] Cor. 4.8) and the above estimate in order to obtain the desired result. The last estimate easily follows by a standard duality argument, combined with the projection estimates of [6] Lemma 4.8, Corollary 4.9; alternatively see [33] Lemma 6.2. Finally, the stability bound on $\left\|u_{\mathrm{ph}}\right\|_{L^{\infty}\left[0, T ; H^{1}(\Omega)\right]}$ is given in [7] Theorem 4.10 .

Remark 4.2. The structure of these estimates for $u-u_{\mathrm{ph}}, v-v_{\mathrm{ph}}$ can be described as follows:

$$
\left.\| \text { error } \|_{X} \leq C \text { ( } \| \text { best approx. error }\left\|_{X}+\right\| \text { init. data error }\left\|_{L^{2}(\Omega)}+\right\| \text { change of subspace error } \|_{X_{1}}\right),
$$

where $X=L^{\infty}\left[0, T ; L^{2}(\Omega)\right] \cap L^{2}\left[0, T ; H_{0}^{1}(\Omega)\right]$. The error due to the change of subspaces is described by the summation term and can be omitted when we use the same subspaces in each time step, i.e. $U_{h}^{n}=U_{h}$. Then, the estimates can be simplified to:

$$
\begin{aligned}
&\left\|u-u_{\mathrm{ph}}\right\|_{L^{2}\left[0, T ; H^{1}(\Omega)\right]} \leq C\left(\|u\|_{L^{2}\left[0, T ; H^{l+1}(\Omega)\right]} h^{l}+\left\|u^{(k+1)}\right\|_{L^{2}\left[0, T ; H^{1}(\Omega)\right]} \tau^{k+1}\right) \\
&\left\|v-v_{\mathrm{ph}}\right\|_{L^{2}\left[0, T ; H^{1}(\Omega)\right]} \leq C\left(\|v\|_{L^{2}\left[0, T ; H^{l+1}(\Omega)\right.} h^{l}+\left\|v^{(k+1)}\right\|_{L^{2}\left[0, T ; H^{1}(\Omega)\right]} \tau^{k+1}\right) .
\end{aligned}
$$

The same simplification also holds for the errors in $L^{\infty}\left[0, T ; L^{2}(\Omega)\right]$ norms.

\subsection{Space-time error estimates}

It remains to bound the error $e_{u h}=u_{\mathrm{ph}}-u_{h}, e_{v h}=v_{\mathrm{ph}}-v_{h}$ in terms of the projection error $e_{u p}=u-u_{\mathrm{ph}}$ and $e_{v p}=v-v_{\mathrm{ph}}$. Then, the error estimates for $e_{u} \equiv u-u_{h}$, and $e_{v} \equiv v-v_{h}$ follow by the triangle inequality and the estimates on $e_{u p}, e_{v p}$. First note that subtracting (3.1)-(3.2) from (2.6)-(2.7) respectively we obtain the following orthogonality condition: for $n=1, \ldots, N$ and for every $w_{h} \in \mathcal{U}_{h}$,

$$
\left(e_{u}^{n}, w_{h}^{n}\right)+\int_{t^{n-1}}^{t^{n}}\left(-\left\langle e_{u}, w_{h t}\right\rangle+a\left(e_{u}, w_{h}\right)+\left(u^{3}-u_{h}^{3}-e_{u}, w_{h}\right)\right) \mathrm{d} t=\left(e_{u}^{n-1}, w_{h+}^{n-1}\right)-\int_{t^{n-1}}^{t^{n}}\left\langle f_{1}-e_{v}, w_{h}\right\rangle \mathrm{d} t
$$

and

$$
\left(e_{v}^{n}, w_{h}^{n}\right)+\int_{t^{n-1}}^{t^{n}}\left(-\left\langle e_{v}, w_{h t}\right\rangle+\delta a\left(e_{v}, w_{h}\right)\right) \mathrm{d} t=\left(e_{v}^{n-1}, w_{h+}^{n-1}\right)+\int_{t^{n-1}}^{t^{n}}\left(\epsilon\left(e_{u}-\alpha_{1} e_{v}, w_{h}\right)\right) \mathrm{d} t .
$$

Here, $f_{1}, f_{2}$ are given data, and $u^{0}, v^{0}$ denote approximations of $u_{0}, v_{0}$ respectively. Splitting the error into $e_{u} \equiv e_{u p}+e_{u h}$ and $e_{v} \equiv e_{v p}+e_{v h}$, using equations (4.1)-(4.2), and adding and subtracting $u_{\mathrm{ph}}^{3}$ in the semilinear 
term, we obtain,

$$
\begin{aligned}
& \left(e_{u h}^{n}, w_{h}^{n}\right)+\int_{t^{n-1}}^{t^{n}}\left(-\left\langle e_{u h}, w_{h t}\right\rangle+a\left(e_{u h}, w_{h}\right)\right) \mathrm{d} t \\
& \quad+\int_{t^{n-1}}^{t^{n}}\left(\left\langle\left(e_{u p}\left(u^{2}+u_{\mathrm{ph}}^{2}+u u_{\mathrm{ph}}\right)+e_{u h}\left(u_{\mathrm{ph}}^{2}+u_{h}^{2}+u_{\mathrm{ph}} u_{h}\right)-\left(e_{u h}+e_{u p}\right), w_{h}\right\rangle\right) \mathrm{d} t\right. \\
& =\left(e_{u h}^{n-1}, w_{h+}^{n-1}\right)+\int_{t^{n-1}}^{t^{n}}-\left\langle e_{v p}+e_{v h}, w_{h}\right\rangle \mathrm{d} t,
\end{aligned}
$$

and

$$
\left(e_{v h}^{n}, w_{h}^{n}\right)+\int_{t^{n-1}}^{t^{n}}\left(-\left\langle e_{v h}, w_{h t}\right\rangle+\delta a\left(e_{v h}, w_{h}\right)\right) \mathrm{d} t=\left(e_{v h}^{n-1}, w_{h+}^{n-1}\right)+\int_{t^{n-1}}^{t^{n}} \epsilon\left(e_{u h}+e_{u p}-\alpha_{1}\left(e_{v p}+e_{v h}\right), w_{h}\right) \mathrm{d} t .
$$

Now, we are ready to prove the bound on $e_{u h}, e_{v h}$ in terms of the parabolic projection of Section 4.1. We will closely follow the arguments of the main stability Proposition 3.4.

Theorem 4.3. Suppose that the assumptions of Proposition 3.4 and Theorem 4.1 hold. In addition, suppose that the time-step $\tau \equiv \max _{i=0, \ldots, n} \tau_{n}$ satisfies $\tau \leq C / C_{\mathrm{st}}^{2}, \tau \leq C_{P} / 8 C_{k}, \tau \leq C \alpha_{1}$ where $C$ denotes a constant depending on $k, \Omega, T$. Here $C_{\mathrm{st}}$, denotes the stability constants of Proposition 3.4. Then, there exists a positive constant $\tilde{C}$ depending upon $k, \Omega, C_{P}$ such that

$$
\begin{aligned}
\int_{0}^{T} & \left(\left\|e_{u h}\right\|_{H^{1}(\Omega)}^{2}+(\delta / \epsilon)\left\|e_{v h}\right\|_{H^{1}(\Omega)}^{2}+\alpha_{1}\left\|e_{v h}\right\|_{L^{2}(\Omega)}^{2}\right) \mathrm{d} t+\left\|e_{u h}^{n}\right\|_{L^{2}(\Omega)}^{2}+(1 / \epsilon)\left\|e_{v h}^{n}\right\|_{L^{2}(\Omega)}^{2} \\
\leq & \tilde{C}\left\{\int_{0}^{T}\left(C_{\mathrm{st}} \tau_{n}^{1 / 2}\left\|e_{u p}\right\|_{H^{1}(\Omega)}^{2}+\left\|e_{v p}\right\|_{H^{1}(\Omega)}^{2}\right) \mathrm{d} t+\int_{0}^{T}\left(\left(1 / \alpha_{1}+1\right)\left\|e_{u p}\right\|_{L^{2}(\Omega)}^{2}+\alpha_{1}\left\|e_{v p}\right\|_{L^{2}(\Omega)}^{2}\right)\right. \\
& \left.+C_{s t, u}^{\prime} \int_{0}^{T}\left\|e_{u p}\right\|_{H^{1}(\Omega)}^{2}\right\} .
\end{aligned}
$$

Here we denote by $e_{u h}=u_{\mathrm{ph}}-u_{h}, e_{v h}=v_{\mathrm{ph}}-v_{h}, e_{u p}=u-u_{\mathrm{ph}}$ and $e_{v p}=v-v_{\mathrm{ph}}$, and $C_{s t, u}^{\prime}$ depends only upon $C_{\mathrm{st}}^{\prime}$ of Theorem 4.1, and $\|u\|_{L^{\infty}\left[0, T ; H^{1}(\Omega)\right]}$.

Proof. Setting $w_{h}=e_{u h}$ into (4.5) we obtain,

$$
\begin{aligned}
& (1 / 2)\left\|e_{u h}^{n}\right\|_{L^{2}(\Omega)}^{2}+\int_{t^{n-1}}^{t^{n}} C_{P}\left\|e_{u h}\right\|_{H^{1}(\Omega)}^{2} \mathrm{~d} t+(1 / 2)\left\|\left[e_{u h}^{n-1}\right]\right\|_{L^{2}(\Omega)}^{2}-(1 / 2)\left\|e_{u h}^{n-1}\right\|_{L^{2}(\Omega)}^{2} \\
& \quad+\int_{t^{n-1}}^{t^{n}}\left(\left(e_{u p}\left(u^{2}+u_{\mathrm{ph}}^{2}+u u_{\mathrm{ph}}\right)+e_{u h}\left(u_{\mathrm{ph}}^{2}+u_{h}^{2}+u_{\mathrm{ph}} u_{h}\right), e_{u h}\right) \mathrm{d} t\right. \\
& \leq-\int_{t^{n-1}}^{t^{n}}\left(e_{v h}, e_{u h}\right) \mathrm{d} t+C \int_{t^{n-1}}^{t^{n}}\left(\left\|e_{u h}\right\|_{L^{2}(\Omega)}^{2}+\left\|e_{u p}\right\|_{L^{2}(\Omega)}^{2}+\left\|e_{v p}\right\|_{L^{2}(\Omega)}^{2}\right) \mathrm{d} t .
\end{aligned}
$$

Here $C$ denotes an algebraic constant. For the nonlinear terms, note that,

$$
\begin{aligned}
& \int_{t^{n-1}}^{t^{n}}\left(\left(e_{u p}\left(u^{2}+u_{\mathrm{ph}}^{2}+u u_{\mathrm{ph}}\right)+e_{u h}\left(u_{\mathrm{ph}}^{2}+u_{h}^{2}+u_{\mathrm{ph}} u_{h}\right), e_{u h}\right) \mathrm{d} t=\int_{t^{n-1}}^{t^{n}} \int_{\Omega} e_{u p}\left(u^{2}+u_{\mathrm{ph}}^{2}+u u_{h p}\right) e_{u h} \mathrm{~d} x \mathrm{~d} t\right. \\
& \quad+\int_{t^{n-1}}^{t^{n}} \int_{\Omega}\left(\left|e_{u h} u_{\mathrm{ph}}\right|^{2}+\left|e_{u h} u_{h}\right|^{2}\right) \mathrm{d} x \mathrm{~d} t+\int_{t^{n-1}}^{t^{n}} \int_{\Omega}\left|e_{u h}\right|^{2} u_{\mathrm{ph}} u_{h} \mathrm{~d} x \mathrm{~d} t .
\end{aligned}
$$


Combining (4.7)-(4.8) we deduce that

$$
\begin{aligned}
& (1 / 2)\left\|e_{u h}^{n}\right\|_{L^{2}(\Omega)}^{2}+\int_{t^{n-1}}^{t^{n}} C_{P}\left\|e_{u h}\right\|_{H^{1}(\Omega)}^{2} \mathrm{~d} t+(1 / 2)\left\|\left[e_{u h}^{n-1}\right]\right\|_{L^{2}(\Omega)}^{2}-(1 / 2)\left\|e_{u h}^{n-1}\right\|_{L^{2}(\Omega)}^{2} \\
& +\int_{t^{n-1}}^{t^{n}}\left(\left\|e_{u h} u_{h}\right\|_{L^{2}(\Omega)}^{2}+\left\|e_{u h} u_{\mathrm{ph}}\right\|_{L^{2}(\Omega)}^{2}\right) \mathrm{d} t \leq-\int_{t^{n-1}}^{t^{n}}\left(e_{v h}, e_{u h}\right) \mathrm{d} t \\
& +\int_{t^{n-1}}^{t^{n}} C\left(\left\|e_{u h}\right\|_{L^{2}(\Omega)}^{2}+\left\|e_{u p}\right\|_{L^{2}(\Omega)}^{2}+\left\|e_{v p}\right\|_{L^{2}(\Omega)}^{2}\right) \mathrm{d} t \\
& +\int_{t^{n-1}}^{t^{n}} \int_{\Omega}\left|e_{u h}\right|^{2}\left|u_{\mathrm{ph}} u_{h}\right| \mathrm{d} x \mathrm{~d} t+\int_{t^{n-1}}^{t^{n}} \int_{\Omega}\left|e_{u p}\left\|u^{2}+u_{\mathrm{ph}}^{2}+u u_{\mathrm{ph}}\right\| e_{u h}\right| \mathrm{d} x \mathrm{~d} t .
\end{aligned}
$$

For the last two integrals, Young's inequality, implies that

$$
\int_{t^{n-1}}^{t^{n}} \int_{\Omega}\left|e_{u h}\right|^{2}\left|u_{\mathrm{ph}} u_{h}\right| \mathrm{d} x \mathrm{~d} t \leq(1 / 2) \int_{t^{n-1}}^{t^{n}}\left\|e_{u h} u_{\mathrm{ph}}\right\|_{L^{2}(\Omega)}^{2} \mathrm{~d} t+(1 / 2) \int_{t^{n-1}}^{t^{n}}\left\|e_{u h} u_{h}\right\|_{L^{2}(\Omega)}^{2} \mathrm{~d} t
$$

while the generalized Cauchy-Schwarz inequality, the embedding $H^{1}(\Omega) \subset L^{4}(\Omega)$, and $\left\|\left|u_{h}\right|^{2}\right\|_{L^{2}(\Omega)} \leq$ $C\left\|u_{h}\right\|_{L^{4}(\Omega)}^{2}$, show that

$$
\begin{aligned}
\int_{t^{n-1}}^{t^{n}} \int_{\Omega}\left|e_{u p}\right|\left|e_{u h}\right|\left|u_{\mathrm{ph}}\right|^{2} \mathrm{~d} x \mathrm{~d} t & \leq \int_{t^{n-1}}^{t^{n}}\left\|e_{u h}\right\|_{L^{4}(\Omega)}\left\|e_{u p}\right\|_{L^{4}(\Omega)}\left\|\left|u_{\mathrm{ph}}\right|^{2}\right\|_{L^{2}(\Omega)} \mathrm{d} t \\
& \leq\left(C_{p} / 8\right) \int_{t^{n-1}}^{t^{n}}\left\|e_{u h}\right\|_{H^{1}(\Omega)}^{2} \mathrm{~d} t+\int_{t^{n-1}}^{t^{n}}\left\|u_{\mathrm{ph}}\right\|_{L^{4}(\Omega)}^{4}\left\|e_{u p}\right\|_{L^{4}(\Omega)}^{2} \mathrm{~d} t \\
& \leq\left(C_{p} / 8\right) \int_{t^{n-1}}^{t^{n}}\left\|e_{u h}\right\|_{H^{1}(\Omega)}^{2} \mathrm{~d} t+C_{\mathrm{st}}^{\prime} \int_{t^{n-1}}^{t^{n}}\left\|e_{u p}\right\|_{H^{1}(\Omega)}^{2} \mathrm{~d} t .
\end{aligned}
$$

The remaining terms can be treated similarly and more easily. Here we have used the stability properties of Proposition 3.4, and Theorem 4.1 for $\left\|u_{\mathrm{ph}}\right\|_{L^{\infty}\left[0, T ; H^{1}(\Omega)\right]} \leq C_{\mathrm{st}}^{\prime}$. Therefore, we deduce from (4.9) the following relation, after dropping positive terms on the left,

$$
\begin{aligned}
& (1 / 2)\left\|e_{u h}^{n}\right\|_{L^{2}(\Omega)}^{2}+\int_{t^{n-1}}^{t^{n}}\left(C_{p} / 8\right)\left\|e_{u h}\right\|_{H^{1}(\Omega)}^{2} \mathrm{~d} t+(1 / 2)\left\|\left[e_{u h}^{n-1}\right]\right\|_{L^{2}(\Omega)}^{2}-(1 / 2)\left\|e_{u h}^{n-1}\right\|_{L^{2}(\Omega)}^{2} \\
& \leq \int_{t^{n-1}}^{t^{n}}-\left(e_{v h}, e_{u h}\right) \mathrm{d} t+C \int_{t^{n-1}}^{t^{n}}\left(\left\|e_{u h}\right\|_{L^{2}(\Omega)}^{2}+\left\|e_{u p}\right\|_{L^{2}(\Omega)}^{2}+\left\|e_{v p}\right\|_{L^{2}(\Omega)}^{2}\right) \mathrm{d} t \\
& \quad+C_{s t, u}^{\prime} \int_{t^{n-1}}^{t^{n}}\left\|e_{u p}\right\|_{H^{1}(\Omega)}^{2} \mathrm{~d} t .
\end{aligned}
$$

Inserting now $v_{h}=e_{v h}$ into (4.6) we easily obtain,

$$
\begin{aligned}
& (1 / 2)\left\|e_{v h}^{n}\right\|_{L^{2}(\Omega)}^{2}+\int_{t^{n-1}}^{t^{n}} \delta\left(C_{P} / 8\right)\left\|e_{v h}\right\|_{H^{1}(\Omega)}^{2} \mathrm{~d} t+(1 / 2)\left\|\left[e_{v h}^{n-1}\right]\right\|_{L^{2}(\Omega)}^{2}-(1 / 2)\left\|e_{v h}^{n-1}\right\|_{L^{2}(\Omega)}^{2} \\
& \quad+\left(\epsilon \alpha_{1} / 4\right) \int_{t^{n-1}}^{t^{n}}\left\|e_{v h}\right\|_{L^{2}(\Omega)}^{2} \mathrm{~d} t \\
& \leq \int_{t^{n-1}}^{t^{n}} \epsilon\left(e_{u h}, e_{v h}\right) \mathrm{d} t+\int_{t^{n-1}}^{t^{n}}\left(\left(\epsilon \alpha_{1} / 4\right)\left\|e_{v p}\right\|_{L^{2}(\Omega)}^{2}+\left(\epsilon / \alpha_{1}\right)\left\|e_{u p}\right\|_{L^{2}(\Omega)}^{2}\right) \mathrm{d} t .
\end{aligned}
$$


Multiplying (4.10) by $\epsilon$ and adding the resulting estimate to (4.11) we deduce,

$$
\begin{aligned}
(1 / 2) \epsilon\left[\left\|e_{u h}^{n}\right\|_{L^{2}(\Omega)}^{2}+\int_{t^{n-1}}^{t^{n}} C_{p} / 4\left\|e_{u h}\right\|_{H^{1}(\Omega)}^{2} \mathrm{~d} t+(1 / 2)\left\|\left[e_{u h}^{n-1}\right]\right\|_{L^{2}(\Omega)}^{2}-(1 / 2)\left\|e_{u h}^{n-1}\right\|_{L^{2}(\Omega)}^{2}\right]+(1 / 2)\left\|e_{v h}^{n}\right\|_{L^{2}(\Omega)}^{2} \\
\quad+\int_{t^{n-1}}^{t^{n}}\left(\delta C_{P} / 8\right)\left\|e_{v h}\right\|_{H^{1}(\Omega)}^{2} \mathrm{~d} t+(1 / 2)\left\|\left[e_{v h}^{n-1}\right]\right\|_{L^{2}(\Omega)}^{2}-(1 / 2)\left\|e_{v h}^{n-1}\right\|_{L^{2}(\Omega)}^{2}+\left(\epsilon \alpha_{1} / 4\right) \int_{t^{n-1}}^{t^{n}}\left\|e_{v h}\right\|_{L^{2}(\Omega)}^{2} \mathrm{~d} t \\
\leq C \int_{t^{n-1}}^{t^{n}}\left(\epsilon\left\|e_{u h}\right\|_{L^{2}(\Omega)}^{2}+\left(\left(\epsilon / \alpha_{1}\right)+\epsilon\right)\left\|e_{u p}\right\|_{L^{2}(\Omega)}^{2}+\left(\epsilon \alpha_{1} / 4\right)\left\|e_{v p}\right\|_{L^{2}(\Omega)}^{2}\right) \mathrm{d} t+\epsilon C_{s t, u}^{\prime} \int_{t^{n-1}}^{t^{n}}\left\|e_{u p}\right\|_{H^{1}(\Omega)}^{2} \mathrm{~d} t .
\end{aligned}
$$

For low order schemes, i.e., for $k=0,1$ it is possible to bound $\int_{t^{n-1}}^{t^{n}}\left\|e_{u h}\right\|_{L^{2}(\Omega)}^{2}$ in terms of the basic energy estimate at the end-points and complete the proof by standard Grönwall type arguments. For high-order schemes we will use the approach of Proposition 3.4. First note that splitting the error $e_{u}=e_{u p}+e_{u h}, e_{v}=e_{v p}+e_{v h}$ into (4.3)-(4.4), and using integration by parts in time, we obtain the analog of (4.5), (switching the integrand variable to $s$,

$$
\begin{aligned}
\int_{t^{n-1}}^{t^{n}}\left(\left\langle\frac{\mathrm{d}}{\mathrm{d} s} e_{u h}, w_{h}\right\rangle+a\left(e_{u h}, w_{h}\right)\right) \mathrm{d} s+\int_{t^{n-1}}^{t^{n}}\left\langle\left( e_{u h}\right.\right. & \left.\left.+e_{u p}\right)\left(u_{h}^{2}+u^{2}+u u_{h}\right), w_{h}\right\rangle \mathrm{d} s \\
& +\left(e_{u h+}^{n-1}-e_{u h}^{n-1}, w_{h+}^{n-1}\right)=\int_{t^{n-1}}^{t^{n}}-\left\langle e_{v p}+e_{v h}, w_{h}\right\rangle \mathrm{d} s .
\end{aligned}
$$

For fixed $t \in\left[t^{n-1}, t^{n}\right)$, we will choose test function $w_{h}=z_{h} \phi(s)$ into (4.13), where $\phi$ is defined similar to Proposition 3.4, and in particular satisfies,

$$
\phi\left(t^{n-1}\right)=1, \quad \int_{t^{n-1}}^{t^{n}} \phi q=\int_{t^{n-1}}^{t} q, \quad q \in \mathcal{P}_{k-1}\left[t^{n-1}, t^{n}\right]
$$

Recall that Lemma 3.3 asserts that $\|\phi\|_{L^{\infty}} \leq C_{k}$, with $C_{k}$ independent of $t$. We note that the above choice of $w_{h}$ leads to

$$
\begin{aligned}
\int_{t^{n-1}}^{t^{n}}\left(\frac{\mathrm{d}}{\mathrm{d} s} e_{u h}, w_{h}\right) \mathrm{d} s+\left(e_{u h+}^{n-1}-e_{u h}^{n-1}, w_{h+}^{n-1}\right) & =\int_{t^{n-1}}^{t}\left(\frac{\mathrm{d}}{\mathrm{d} s} e_{u h}, z_{h}\right) \mathrm{d} s+\left(e_{u h+}^{n-1}-e_{u h}^{n-1}, \phi\left(t^{n-1}\right) z_{h}\right) \\
& =\left(e_{u h}(t)-e_{u h}^{n-1}, z_{h}\right) .
\end{aligned}
$$

Hence substituting the last relation into (4.13) we obtain

$$
\begin{aligned}
\left(e_{u h}(t)-e_{u h}^{n-1}, z_{h}\right)= & -\int_{t^{n-1}}^{t^{n}}\left(a\left(e_{u h}, z_{h}\right)+\left(\left(e_{u h}+e_{u p}\right)\left(u_{h}^{2}+u^{2}+u_{h} u\right), z_{h}\right)\right) \phi \mathrm{d} s+\int_{t^{n-1}}^{t^{n}}-\left(e_{u h}+e_{v h}, z_{h}\right) \phi \mathrm{d} s \\
\leq & C_{k}\left[\int_{t^{n-1}}^{t^{n}}\left(\left\|\nabla e_{u h}\right\|_{L^{2}(\Omega)}\left\|\nabla z_{h}\right\|_{L^{2}(\Omega)}+\left\|e_{u h}\right\|_{L^{2}(\Omega)}\left\|z_{h}\right\|_{L^{2}(\Omega)}\right) \mathrm{d} s\right. \\
& +\int_{t^{n-1}}^{t^{n}}\left(\left\|e_{u h}\right\|_{L^{4}(\Omega)}\left(\left\|u_{h}^{2}\right\|_{L^{2}(\Omega)}+\left\|u^{2}\right\|_{L^{2}(\Omega)}\right)\left\|z_{h}\right\|_{L^{4}(\Omega)}\right) \mathrm{d} s \\
& +\int_{t^{n-1}}^{t^{n}}\left(\left\|e_{u p}\right\|_{L^{4}(\Omega)}\left(\left\|u_{h}^{2}\right\|_{L^{2}(\Omega)}+\left\|u^{2}\right\|_{L^{2}(\Omega)}\right)\left\|z_{h}\right\|_{L^{4}(\Omega)}\right) \mathrm{d} s \\
& \left.+\int_{t^{n-1}}^{t^{n}}\left\|e_{v h}\right\|_{L^{2}(\Omega)}\left\|z_{h}\right\|_{H^{1}(\Omega)} \mathrm{d} s\right]
\end{aligned}
$$


We need to bound the terms on the left. Note that $\left\|u_{h}^{2}\right\|_{L^{2}(\Omega)} \leq C\left\|u_{h}\right\|_{L^{4}(\Omega)}^{2}$ and that $z_{h}$ is chosen independent of $t$. Hence,

$$
\begin{aligned}
\int_{t^{n-1}}^{t^{n}}\left\|e_{u h}\right\|_{L^{4}(\Omega)}\left\|u_{h}^{2}\right\|_{L^{2}(\Omega)}\left\|z_{h}\right\|_{L^{4}(\Omega)} \mathrm{d} s & \leq\left(\int_{t^{n-1}}^{t^{n}}\left\|e_{u h}\right\|_{L^{4}(\Omega)}\left\|u_{h}\right\|_{L^{4}(\Omega)}^{2} \mathrm{~d} s\right)\left\|z_{h}\right\|_{L^{4}(\Omega)} \\
& \leq\left(\int_{t^{n-1}}^{t^{n}}\left\|e_{u h}\right\|_{H^{1}(\Omega)}\left\|u_{h}\right\|_{L^{2}(\Omega)}\left\|u_{h}\right\|_{H^{1}(\Omega)} \mathrm{d} s\right)\left\|z_{h}\right\|_{H^{1}(\Omega)} \\
& \leq C_{\mathrm{st}}\left\|e_{u h}\right\|_{L^{2}\left[t^{n-1}, t^{n} ; H^{1}(\Omega)\right]}\left\|z_{h}\right\|_{H^{1}(\Omega)},
\end{aligned}
$$

where we have used the Gagliardo-Nirenberg inequality and the stability estimate on $u_{h}$ at arbitrary time-points first, and then the Cauchy-Schwarz inequality. The remaining nonlinear terms can be handled similarly. For the linear terms, we note that,

$$
\int_{t^{n-1}}^{t^{n}}\left\|\nabla e_{u h}\right\|_{L^{2}(\Omega)}\left\|\nabla z_{h}\right\|_{L^{2}(\Omega)} \mathrm{d} s \leq C \tau_{n}^{1 / 2}\left\|e_{u h}\right\|_{L^{2}\left[t^{n-1}, t^{n} ; H^{1}(\Omega)\right]}\left\|z_{h}\right\|_{H^{1}(\Omega)} .
$$

The rest of terms can be handled similarly. Hence, we obtain

$$
\begin{aligned}
\left(e_{u h}(t)-e_{u h}^{n-1}, z_{h}\right) \leq & C_{k}\left[\tau_{n}^{1 / 2}\left\|e_{u h}\right\|_{L^{2}\left[t^{n-1}, t^{n} ; H^{1}(\Omega)\right]}+\tau_{n}^{1 / 2}\left\|e_{v h}\right\|_{L^{2}\left[t^{n-1}, t^{n} ; L^{2}(\Omega)\right]}\right]\left\|z_{h}\right\|_{H^{1}(\Omega)} \\
& +C_{k} C_{\mathrm{st}}\left[\left\|e_{u h}\right\|_{L^{2}\left[t^{n-1}, t^{n} ; H^{1}(\Omega)\right]}+\left\|e_{u p}\right\|_{L^{2}\left[t^{n-1}, t^{n} ; H^{1}(\Omega)\right]}\left\|z_{h}\right\|_{H^{1}(\Omega)} .\right.
\end{aligned}
$$

Selecting now $z_{h}=e_{u h}(t)$ and integrating with respect to $t$ the above inequality, and using Cauchy-Schwarz once more we deduce a bound on $\left\|e_{u h}\right\|_{L^{2}\left[0, T ; L^{2}(\Omega)\right]}^{2}$, i.e.,

$$
\begin{aligned}
\int_{t^{n-1}}^{t^{n}}\left\|e_{u h}\right\|_{L^{2}(\Omega)}^{2} \mathrm{~d} t \leq \tau_{n}\left\|e_{u h}^{n-1}\right\|_{L^{2}(\Omega)}^{2}+C_{k} \tau_{n} \int_{t^{n-1}}^{t^{n}}\left(\left\|e_{u h}\right\|_{H^{1}(\Omega)}^{2}+\left\|e_{v h}\right\|_{L^{2}(\Omega)}^{2}\right) \mathrm{d} t \\
+C_{k} C_{\mathrm{st}} \tau_{n}^{1 / 2} \int_{t^{n-1}}^{t^{n}}\left(\left\|e_{u h}\right\|_{H^{1}(\Omega)}^{2}+\left\|e_{u p}\right\|_{H^{1}(\Omega)}^{2}\right) \mathrm{d} t .
\end{aligned}
$$

The proof now follows identical to [7] Theorem 5.2, after noting that we may combine (4.14) to replace $\int_{t^{n-1}}^{t^{n}}\left\|e_{u h}\right\|_{L^{2}(\Omega)}^{2} \mathrm{~d} t$ from (4.12) and choose $\tau_{n}$ in such a way to hide the $\int_{t^{n-1}}^{t^{n}}\left\|e_{u h}\right\|_{H^{1}(\Omega)}^{2} \mathrm{~d} t$ and $\int_{t^{n-1}}^{t^{n}}\left\|e_{v h}\right\|_{L^{2}(\Omega)}^{2} \mathrm{~d} t$ on the left hand side, i.e., we choose $\tau_{n}$ such that

$$
\left.\left(C_{P} \epsilon / 2\right)-\tau_{n}^{1 / 2} \epsilon C_{k} C_{\mathrm{st}}\right) \geq\left(C_{p} \epsilon / 8\right), \quad \tau_{n} \epsilon C_{k} \leq\left(C_{p} \epsilon / 8\right), \text { and } \quad \epsilon \alpha_{1} / 4-\tau_{n} \epsilon C_{k} \geq\left(\epsilon \alpha_{1} / 8\right) .
$$

Therefore, we obtain,

$$
\begin{aligned}
& (1 / 2) \epsilon\left[\left\|e_{u h}^{n}\right\|_{L^{2}(\Omega)}^{2}+\int_{t^{n-1}}^{t^{n}}\left(C_{p} / 4\right)\left\|e_{u h}\right\|_{H^{1}(\Omega)}^{2} \mathrm{~d} t+(1 / 2)\left\|\left[e_{u h}^{n-1}\right]\right\|_{L^{2}(\Omega)}^{2}\right]+(1 / 2)\left\|e_{v h}^{n}\right\|_{L^{2}(\Omega)}^{2} \\
& \quad+\int_{t^{n-1}}^{t^{n}}\left(\delta C_{P} / 8\right)\left\|e_{v h}\right\|_{H^{1}(\Omega)}^{2} \mathrm{~d} t+(1 / 2)\left\|\left[e_{v h}^{n-1}\right]\right\|_{L^{2}(\Omega)}^{2}+\left(\epsilon \alpha_{1} / 8\right) \int_{t^{n-1}}^{t^{n}}\left\|e_{v h}\right\|_{L^{2}(\Omega)}^{2} \mathrm{~d} t \\
& \leq \epsilon\left(1 / 2+\tau_{n}\right)\left\|e_{u h}^{n-1}\right\|_{L^{2}(\Omega)}^{2}+(1 / 2)\left\|e_{v h}^{n-1}\right\|_{L^{2}(\Omega)}^{2} \\
& \quad+\int_{t^{n-1}}^{t^{n}}\left(\left(\left(\epsilon / \alpha_{1}\right)+\epsilon\right)\left\|e_{u p}\right\|_{L^{2}(\Omega)}^{2}+\left(\epsilon \alpha_{1} / 4+\epsilon\right)\left\|e_{v p}\right\|_{L^{2}(\Omega)}^{2}\right) \mathrm{d} t \\
& +\epsilon\left(C_{\mathrm{st}} \tau_{n}^{1 / 2}+C_{s t, u}^{\prime}\right) \int_{t^{n-1}}^{t^{n}}\left\|e_{u p}\right\|_{H^{1}(\Omega)}^{2} \mathrm{~d} t
\end{aligned}
$$

A discrete Grönwal lemma finishes the proof. 
TABLE 1. Rates of convergence for the $1 \mathrm{~d}$ solution with $l=1, k=0, \epsilon=10^{-3}$.

\begin{tabular}{|c|c|c|c|c|}
\hline$h=\tau$ & $\left\|e_{u}\right\|_{L^{2}\left[0, T ; H^{1}(\Omega)\right]}$ & Rate & $\left\|e_{v}\right\|_{L^{2}\left[0, T ; H^{1}(\Omega)\right]}$ & Rate \\
\hline $1 / 4$ & $8.567888 \mathrm{e}-02$ & & $3.346261 \mathrm{e}-03$ & \\
$1 / 8$ & $4.286834 \mathrm{e}-02$ & $5.912449 \mathrm{e}-01$ & $2.844631 \mathrm{e}-03$ & $2.343084 \mathrm{e}-01$ \\
$1 / 16$ & $2.143254 \mathrm{e}-02$ & $6.471675 \mathrm{e}-01$ & $2.272942 \mathrm{e}-03$ & $3.236805 \mathrm{e}-01$ \\
$1 / 32$ & $1.072671 \mathrm{e}-02$ & $7.264316 \mathrm{e}-01$ & $1.521046 \mathrm{e}-03$ & $5.794969 \mathrm{e}-01$ \\
$1 / 64$ & $5.383031 \mathrm{e}-03$ & $8.110452 \mathrm{e}-01$ & $7.981191 \mathrm{e}-04$ & $9.303881 \mathrm{e}-01$ \\
$1 / 128$ & $2.720939 \mathrm{e}-03$ & $8.753305 \mathrm{e}-01$ & $4.013479 \mathrm{e}-04$ & $9.917505 \mathrm{e}-01$ \\
\hline Norm & $3.219971 \mathrm{e}-01$ & & $4.161678 \mathrm{e}-03$ & \\
\hline
\end{tabular}

Remark 4.4. Note that the restriction on the time step depends mildly upon the $\epsilon$.

Combining the estimates of the previous theorem together with estimates on the parabolic projections of Section 4 , we deduce rates of convergence. Below, we state the relevant rates for smooth solutions $u, v$ as well as rate for the case $U_{h}^{n}=U_{h}$ for $n=1, \ldots, N$.

Theorem 4.5. Suppose that the assumptions of Proposition 3.4 and Theorems 4.1, 4.3 hold. Then for $u, v \in$ $C\left[0, T ; H^{l+1}(\Omega)\right] \cap H^{k+1}\left[0, T ; H_{0}^{1}(\Omega)\right]$ the following estimate holds:

$$
\begin{aligned}
\left\|e_{u}\right\|_{L^{2}\left[0, T ; H^{1}(\Omega)\right]}+\sqrt{\delta / \epsilon}\left\|e_{v}\right\|_{L^{2}\left[0, T ; H^{1}(\Omega)\right]} \leq \tilde{C}\left\{\|u\|_{C\left[0, T ; H^{l+1}(\Omega)\right]} h^{l}+\left\|u^{(k+1)}\right\|_{L^{2}\left[0, T ; H^{1}(\Omega)\right]} \tau^{k+1}\right. \\
+\|u\|_{C\left[0, T ; H^{l+1}(\Omega)\right]} \min \left(h^{2} / \tau, h / \sqrt{\tau}\right) h^{l}+\|v\|_{L^{2}\left[0, T ; H^{l+1}(\Omega)\right]} h^{l} \\
\left.+\left\|v^{(k+1)}\right\|_{L^{2}\left[0, T ; H^{1}(\Omega)\right]} \tau^{k+1}+\|v\|_{C\left[0, T ; H^{l+1}(\Omega)\right]} \min \left(h^{2} /(\tau \delta), h / \sqrt{\tau \delta}\right) h^{l}\right\} .
\end{aligned}
$$

If in addition the same subspaces are being used every time-step, i.e., $U_{h}^{n}=U_{h}$ for $n=1, \ldots, N$, then

$$
\begin{aligned}
\left\|e_{u}\right\|_{L^{2}\left[0, T ; H^{1}(\Omega)\right]}+\sqrt{\delta / \epsilon}\left\|e_{v}\right\|_{L^{2}\left[0, T ; H^{1}(\Omega)\right]} \leq & \tilde{C}\left\{\|u\|_{C\left[0, T ; H^{l+1}(\Omega)\right]} h^{l}+\left\|u^{(k+1)}\right\|_{L^{2}\left[0, T ; H^{1}(\Omega)\right]} \tau^{k+1}\right. \\
& \left.+\|v\|_{L^{2}\left[0, T ; H^{l+1}(\Omega)\right]} h^{l}+\left\|v^{(k+1)}\right\|_{L^{2}\left[0, T ; H^{1}(\Omega)\right]} \tau^{k+1}\right\} .
\end{aligned}
$$

Proof. The proof follows by combining Theorems 4.3, 4.1 and triangle inequality.

\section{NumERICAL EXPERIMENTS}

In this section we present some numerical experiments of the proposed methodology.

\subsection{Example 1: 1-D problem with smooth initial values}

First, we are concerned in measuring the $L^{2}\left[0, T ; H^{1}(\Omega)\right]$ norms for both $u, v$ variables, in case that $d=1$ and piecewise linear polynomials in space $(l=1)$ and $k=0,1,2$ polynomials in time are being used. For the choice of parameters $\delta=4, \alpha_{1}=2$ (similar to [39]) we set $\epsilon=0.001$. The external forces $f_{1}, f_{2}$ are chosen in a way to guarantee that $u(t, x)=\frac{t}{1+t^{2}} \sin \left(\frac{\pi x}{2}\right)(1-x), v(t, x)=\frac{\epsilon t}{\epsilon+t^{2}} \cos \left(\frac{\pi x}{2}\right) x$ are solutions of the FHN system in the interval $[0,1] \times[0,1]$.

It is clear that $v$ attains its maximum value $v_{\max }=\sqrt{2 \epsilon} / 8$ at $t=\sqrt{\epsilon}$ and then decays to zero for large $t$. On the other hand, $u$ rises slowly, reaching its maximum $\sqrt{2} / 8$ at $t=1$ and thus, the selected functions scale significantly different in time for small $\epsilon$. Thus, one should expect poor performance of the relevant approximate solution when coarse grids in time are to be employed in combination with small $\epsilon$ values. In Tables $1-3$ we present the errors in the $L^{2}\left[0, T ; H^{1}(\Omega)\right]$ norm for $\epsilon=0.001$ and the relevant rates of convergence, as they result with grid refinement for $k=0,1,2$ respectively. In the ultimate line we denote the values of the $L^{2}\left[0, T ; H^{1}(\Omega)\right]$ 
TABLE 2. Rates of convergence for the $1 \mathrm{~d}$ solution with $l=1, k=1, \epsilon=10^{-3}$.

\begin{tabular}{|c|c|c|c|c|}
\hline$h=\tau$ & $\left\|e_{u}\right\|_{L^{2}\left[0, T ; H^{1}(\Omega)\right]}$ & Rate & $\left\|e_{v}\right\|_{L^{2}\left[0, T ; H^{1}(\Omega)\right]}$ & Rate \\
\hline $1 / 4$ & $7.637934 \mathrm{e}-02$ & & $1.845876 \mathrm{e}-03$ & \\
$1 / 8$ & $3.809843 \mathrm{e}-02$ & $1.003451 \mathrm{e} 00$ & $1.286796 \mathrm{e}-03$ & $5.205216 \mathrm{e}-01$ \\
$1 / 16$ & $1.904048 \mathrm{e}-02$ & $1.000662 \mathrm{e} 00$ & $7.450850 \mathrm{e}-04$ & $7.883068 \mathrm{e}-01$ \\
$1 / 32$ & $9.523825 \mathrm{e}-03$ & $9.994572 \mathrm{e}-01$ & $3.388446 \mathrm{e}-03$ & $1.136781 \mathrm{e} 00$ \\
$1 / 64$ & $4.771371 \mathrm{e}-03$ & $9.971372 \mathrm{e}-01$ & $1.008715 \mathrm{e}-04$ & $1.748106 \mathrm{e} 00$ \\
$1 / 128$ & $2.404897 \mathrm{e}-03$ & $9.884286 \mathrm{e}-01$ & $3.634394 \mathrm{e}-05$ & $1.472731 \mathrm{e} 00$ \\
\hline Norm & $3.219971 \mathrm{e}-01$ & & $4.161678 \mathrm{e}-03$ & \\
\hline
\end{tabular}

TABLE 3. Rates of convergence for the $1 \mathrm{~d}$ solution with $l=1, k=2, \epsilon=10^{-3}$.

\begin{tabular}{|c|c|c|c|c|}
\hline$h=\tau$ & $\left\|e_{u}\right\|_{L^{2}\left[0, T ; H^{1}(\Omega)\right]}$ & Rate & $\left\|e_{v}\right\|_{L^{2}\left[0, T ; H^{1}(\Omega)\right]}$ & Rate \\
\hline $1 / 4$ & $7.629455 \mathrm{e}-02$ & & $1.582274 \mathrm{e}-03$ & \\
$1 / 8$ & $3.808815 \mathrm{e}-02$ & $1.002238 \mathrm{e} 00$ & $9.397418 \mathrm{e}-04$ & $7.516631 \mathrm{e}-01$ \\
$1 / 16$ & $1.903925 \mathrm{e}-02$ & $1.000365 \mathrm{e} 00$ & $4.088469 \mathrm{e}-04$ & $1.200704 \mathrm{e} 00$ \\
$1 / 32$ & $9.523531 \mathrm{e}-03$ & $9.994084 \mathrm{e}-01$ & $1.489608 \mathrm{e}-04$ & $1.456628 \mathrm{e} 00$ \\
$1 / 64$ & $4.771323 \mathrm{e}-03$ & $9.971073 \mathrm{e}-01$ & $6.901361 \mathrm{e}-05$ & $1.109980 \mathrm{e} 00$ \\
$1 / 128$ & $2.404888 \mathrm{e}-03$ & $9.884195 \mathrm{e}-01$ & $3.371538 \mathrm{e}-05$ & $1.033474 \mathrm{e} 00$ \\
\hline Norm & $3.219971 \mathrm{e}-01$ & & $4.161678 \mathrm{e}-03$ & \\
\hline
\end{tabular}

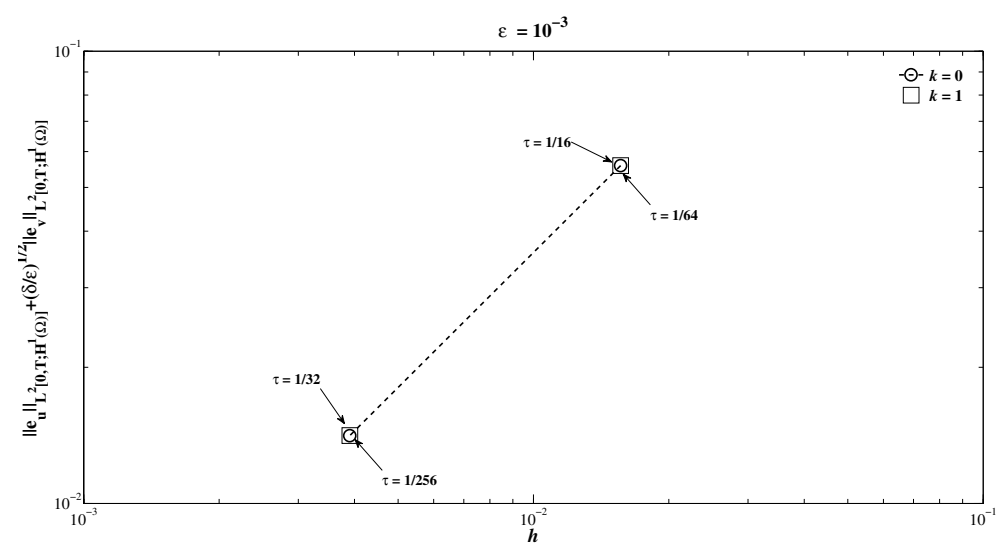

Figure 1. Convergence in the compound norm for $k=0$ and $k=1$ with different time steps.

norms of the exact solution pair. It is clear that the rates are almost identical to one, which is in agreement with the estimation of Theorem 4.5 , since $l=1$ for all cases. Note that the required error norms were computed using the Gauss-Legendre rule with $n=10$.

To demonstrate the validity of the error estimation presented in Theorem 4.5, we have compared the error from methods of different order in time, employing the same grid in space and an accordingly coarser grid in time. More specifically, we have calculated the error in the compound norm $\left\|e_{u}\right\|_{L^{2}\left[0, T ; H^{1}(\Omega)\right.}+\sqrt{\delta / \epsilon}\left\|e_{v}\right\|_{L^{2}\left[0, T ; H^{1}(\Omega)\right]}$ as it yields from the lower order in time $(k=0)$ method with time step size $\tau_{0}=1 / 64$ and $1 / 256, h_{0}=\tau_{0}$ and the same quantity produced by the higher order in time $(k=1)$ method with corresponding time step size $\tau_{1}=2 \sqrt{\tau_{0}}$ and $h_{1}=h_{0}=\tau_{0}$. The same comparison has been done for the method with $k=1$ using $h_{1}=\tau_{1}=1 / 64,1 / 128$ and $1 / 256$ and for the method with $k=2$ using $\tau_{2}=2\left\lfloor\tau_{1}^{2 / 3}\right\rfloor$ and $h_{2}=h_{1}$. The results are shown in Figures 1 and 2, by which it is evident that the order of convergence in $\tau$ estimated by (4.17) is verified. 


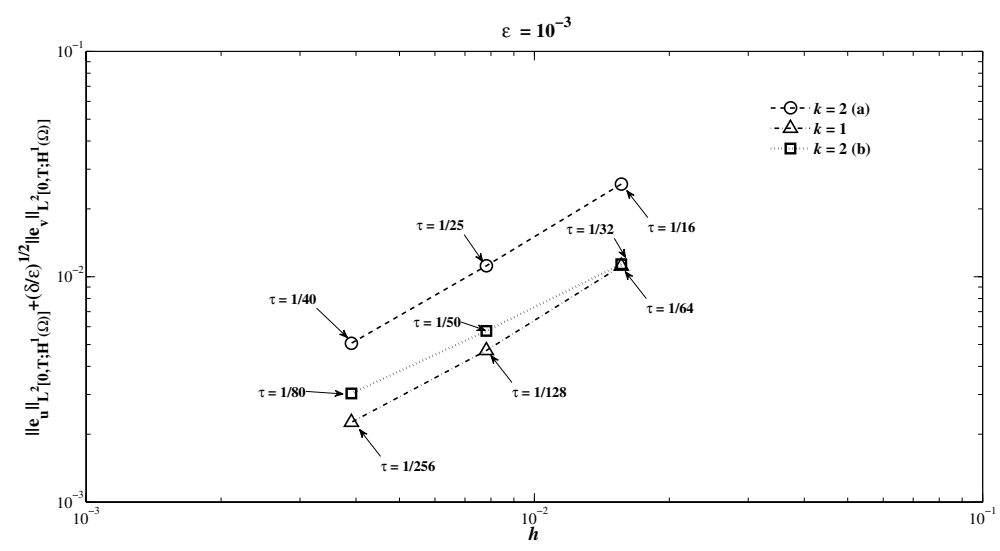

FIgURE 2. Convergence in the compound norm for $k=1$ and $k=2$ with different time steps.
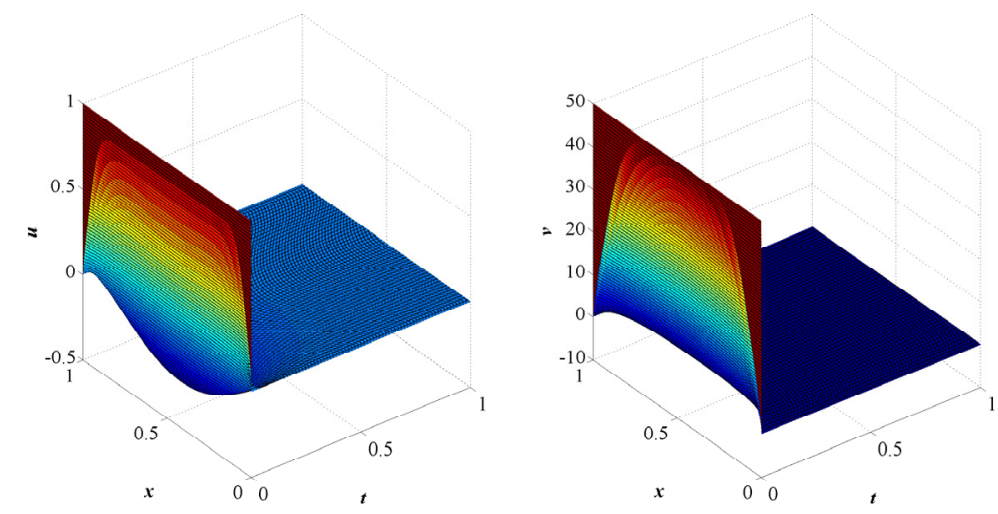

Figure 3. Plots of $u_{h}(x, t)$ and $v_{h}(x, t)$ for the second example.

\subsection{Example 2: 1-D problem with $L^{2}(\Omega)$ initial values}

In the second experiment, we evolve the system with initial data given by $u_{0}=1$ and $v_{0}=\frac{1}{\epsilon \alpha_{1}}$ and $f_{1}=f_{2} \equiv 0$. Then, for the same set of parameters, $\delta, \alpha_{1}$ and for $\epsilon=0.01$ we compute by the discontinuous time-stepping scheme for $k=0,1,2, l=1$, in the interval $[0,1] \times\left[0, \frac{1}{2}\right]$. It is obvious, in this case, that $u_{0}, v_{0} \in L^{2}(0,1)$. In Figure 3 we show the graphs of $u(x, t)$ and $v(x, t)$ as computed using $l=1, k=2, \tau=h=\frac{1}{512}$ which is considered the reference solution. Our aim is to verify that the higher order method $(k=1)$ yields comparable error in the appropriate norm as the lower order method $(k=0)$ by keeping the same grid in space and using a much coarser grid in time, i.e. taking $\tau_{1}=\left(\tau_{0}\right)^{\frac{1}{2}}$, where $\tau_{1}$ is the grid size for $k=1$ and $\tau_{0}$ is the corresponding quantity for $k=0$. To this end, we evolve the system for $l=1, k=0, h_{0}=\tau_{0}=1 / 16,1 / 64,1 / 256$ and for $k=1, h_{1}=h_{0}, \tau_{1}=\sqrt{\tau_{0}}$, calculating the error norms with respect to the reference solution. In Figure 4 we present the evolution of the error as a function of spatial grid size with the time step as a parameter for both low order $(k=0)$ and high order $(k=1)$ methods. We observe that there is a decay in the size of the error in the $L^{2}\left[0, T ; L^{2}(\Omega)\right]$ norm with mesh refinement as predicted by Theorem 3.8. Further, for the same $h$, the high order method manages to produce an error in the same order of magnitude with that of the low order method, employing a significantly coarser temporal grid $\left(\tau_{1}=\sqrt{\tau_{0}}\right)$. 


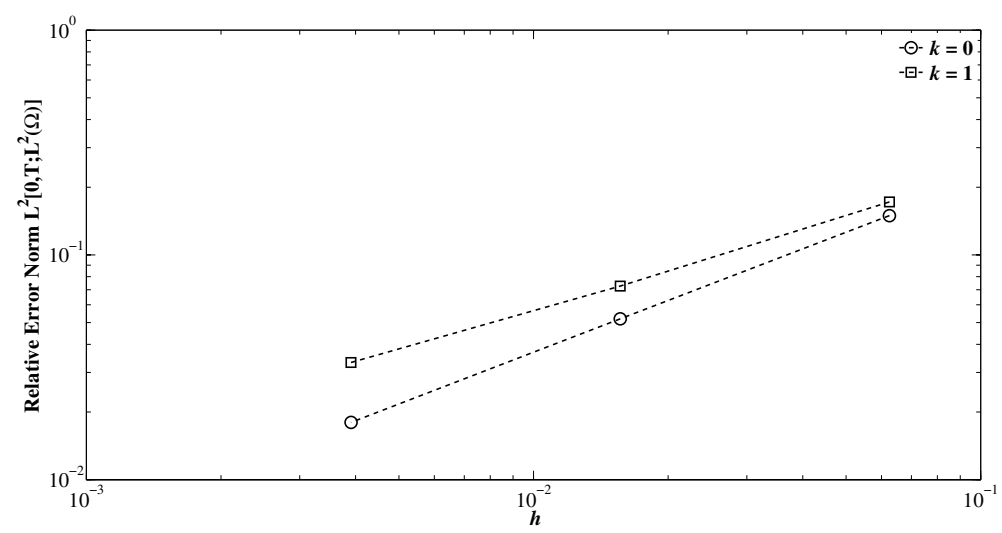

Figure 4. Convergence in the $L^{2}\left[0, T ; L^{2}(\Omega)\right]$ norm for $k=0$ and $k=1$ for the second example.
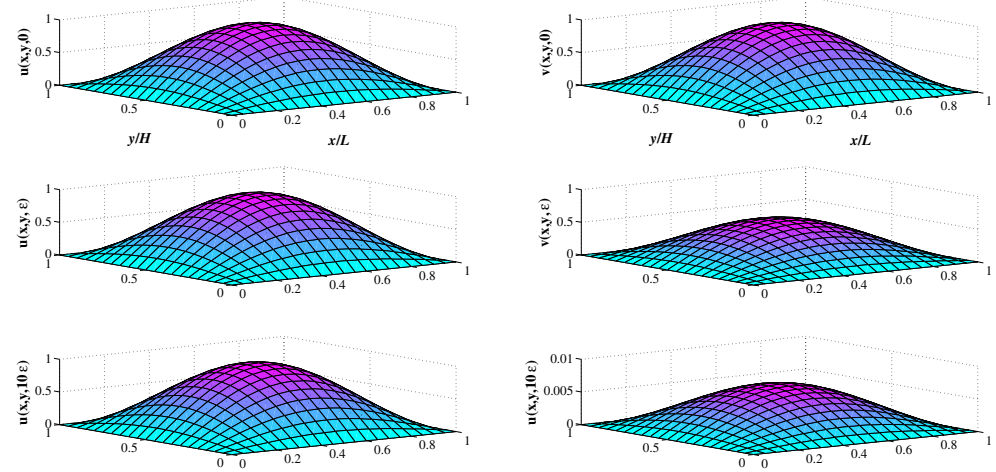

Figure 5. Plots of exact solution pair for the second example and $t=0, \epsilon, 10 \epsilon$.

TABLE 4. Rates of convergence for the second solution with $l=1, k=0, \epsilon=10^{-3}$.

\begin{tabular}{|c|c|c|c|c|}
\hline$h=\tau$ & $\left\|e_{u}\right\|_{L^{2}\left[0, T ; H^{1}(\Omega)\right]}$ & Rate & $\left\|e_{v}\right\|_{L^{2}\left[0, T ; H^{1}(\Omega)\right]}$ & Rate \\
\hline $1 / 4$ & $8.423625 \mathrm{e}-02$ & & $4.670405 \mathrm{e}-02$ & \\
$1 / 8$ & $4.322326 \mathrm{e}-02$ & $9.626333 \mathrm{e}-01$ & $2.668618 \mathrm{e}-02$ & $8.074546 \mathrm{e}-01$ \\
$1 / 16$ & $2.175905 \mathrm{e}-02$ & $9.901927 \mathrm{e}-01$ & $1.398540 \mathrm{e}-02$ & $9.321719 \mathrm{e}-01$ \\
$1 / 32$ & $1.089823 \mathrm{e}-03$ & $9.975214 \mathrm{e}-01$ & $7.116571 \mathrm{e}-03$ & $9.746669 \mathrm{e}-01$ \\
\hline Norm & $2.221436 \mathrm{e}-01$ & & $7.024673 \mathrm{e}-02$ & \\
\hline
\end{tabular}

\subsection{Example 3: A 2-D problem}

In this example we consider the performance of the numerical method for a problem having the following solution: $u(x, y, t)=\mathrm{e}^{-\epsilon t} \sin \left(\frac{\pi x}{L}\right) \sin \left(\frac{\pi y}{H}\right), v(x, y, t)=\mathrm{e}^{-t /(2 \epsilon)} \sin \left(\frac{\pi x}{L}\right) \sin \left(\frac{\pi y}{H}\right)$. The physical domain dimensions are $L=H=0.01$ and the time interval length $T=0.01$. In Figure 5 we show a plot of the solution for $t=0, \epsilon, 10 \epsilon$ and for $\epsilon=10^{-4}$. It is obvious that, while $u$ remains practically constant, $v$ decays rapidly. For the discretization in space we have used standard Galerkin formulation and linear triangular elements with 2 degrees of freedom per node, while for time discretization $k=0,1$ has been employed. We chose $\delta=4, \alpha_{1}=2$ for the parameter values and compute for the cases $\epsilon=10^{-3}$ and $\epsilon=10^{-4}$. In Tables 4 and 5 we present the errors in the $L^{2}\left[0, T ; H^{1}(\Omega)\right]$ norm for $\epsilon=10^{-3}$ along with the respective rates of convergence for $k=0,1$. Similarly, 
TABLE 5. Rates of convergence for the second solution with $l=1, k=1, \epsilon=10^{-3}$.

\begin{tabular}{|c|c|c|c|c|}
\hline$h=\tau$ & $\left\|e_{u}\right\|_{L^{2}\left[0, T ; H^{1}(\Omega)\right]}$ & Rate & $\left\|e_{v}\right\|_{L^{2}\left[0, T ; H^{1}(\Omega)\right]}$ & Rate \\
\hline $1 / 4$ & $8.421848 \mathrm{e}-02$ & & $2.732333 \mathrm{e}-02$ & \\
$1 / 8$ & $4.322319 \mathrm{e}-02$ & $9.626331 \mathrm{e}-01$ & $1.376980 \mathrm{e}-02$ & $9.886259 \mathrm{e}-01$ \\
$1 / 16$ & $2.175904 \mathrm{e}-02$ & $9.901909 \mathrm{e}-01$ & $6.893596 \mathrm{e}-03$ & $9.981786 \mathrm{e}-01$ \\
$1 / 32$ & $1.089823 \mathrm{e}-03$ & $9.975214 \mathrm{e}-01$ & $3.447845 \mathrm{e}-03$ & $9.995618 \mathrm{e}-01$ \\
\hline Norm & $2.221436 \mathrm{e}-01$ & & $7.024673 \mathrm{e}-02$ & \\
\hline
\end{tabular}

TABLE 6. Rates of convergence for the second solution with $l=1, k=0, \epsilon=10^{-4}$.

\begin{tabular}{|c|c|c|c|c|}
\hline$h=4 \tau$ & $\left\|e_{u}\right\|_{L^{2}\left[0, T ; H^{1}(\Omega)\right]}$ & Rate & $\left\|e_{v}\right\|_{L^{2}\left[0, T ; H^{1}(\Omega)\right.}$ & Rate \\
\hline $1 / 4$ & $8.421064 \mathrm{e}-02$ & & $2.067186 \mathrm{e}-02$ & \\
$1 / 8$ & $4.322338 \mathrm{e}-02$ & $9.621906 \mathrm{e}-01$ & $1.524974 \mathrm{e}-02$ & $4.388833 \mathrm{e}-01$ \\
$1 / 16$ & $2.175914 \mathrm{e}-02$ & $9.901902 \mathrm{e}-01$ & $9.045178 \mathrm{e}-03$ & $7.533639 \mathrm{e}-01$ \\
$1 / 32$ & $1.089828 \mathrm{e}-03$ & $9.975214 \mathrm{e}-01$ & $4.841640 \mathrm{e}-03$ & $9.016530 \mathrm{e}-01$ \\
\hline Norm & $2.221436 \mathrm{e}-01$ & & $2.221447 \mathrm{e}-02$ & \\
\hline
\end{tabular}

TABLE 7. Rates of convergence for the second solution with $l=1, k=1, \epsilon=10^{-4}$.

\begin{tabular}{|c|c|c|c|c|}
\hline$h=\tau$ & $\left\|e_{u}\right\|_{L^{2}\left[0, T ; H^{1}(\Omega)\right]}$ & Rate & $\left\|e_{v}\right\|_{L^{2}\left[0, T ; H^{1}(\Omega)\right]}$ & Rate \\
\hline $1 / 4$ & $8.421886 \mathrm{e}-02$ & & $1.857317 \mathrm{e}-02$ & \\
$1 / 8$ & $4.322338 \mathrm{e}-02$ & $9.623314 \mathrm{e}-01$ & $1.596766 \mathrm{e}-02$ & $2.180675 \mathrm{e}-01$ \\
$1 / 16$ & $2.175913 \mathrm{e}-02$ & $9.901909 \mathrm{e}-01$ & $8.245388 \mathrm{e}-03$ & $9.534935 \mathrm{e}-01$ \\
$1 / 32$ & $1.089827 \mathrm{e}-03$ & $9.975214 \mathrm{e}-01$ & $2.973031 \mathrm{e}-03$ & $1.471653 \mathrm{e} 00$ \\
\hline Norm 0 & $2.221436 \mathrm{e}-01$ & & $2.221447 \mathrm{e}-02$ & \\
\hline
\end{tabular}

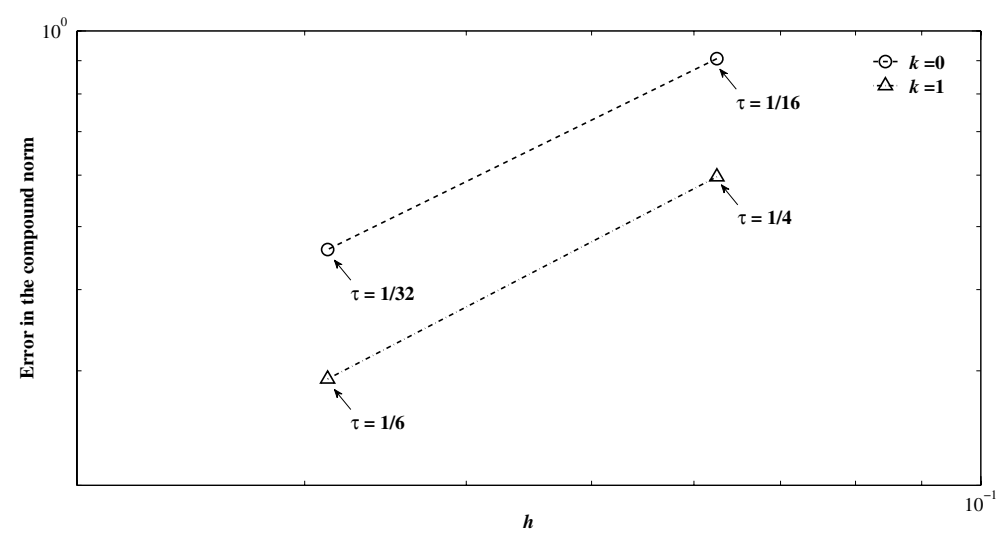

FiguRE 6 . Convergence in the compound norm for $k=0$ and $k=1$ for the second example.

in Tables 6 and 7, we present the same quantities for $\epsilon=10^{-4}$. In all cases we use $\tau \approx h$ and we observe the predicted rates for $u$, and $v$, while for $\epsilon=10^{-4}$ the exact predicted rate for $v$ follows upon further refinement. If we have chosen exact solution pair with time derivatives varying moderately in size with respect to $\epsilon$, then theoretical predictions would have been easily verified. However, even if the chosen example is better suited for adaptive machineries, the proposed method manages to cope with the relatively coarse grids employed. Finally, to demonstrate the ability of the higher order methods to perform equally well employing coarser grids in time, we present in Figure 6 the comparative results for the error in the compound norm for $\epsilon=10^{-3}$ and $k=0,1$. We employed 2 grids for $k=0$, i.e. one with $h=\tau=1 / 16$ and one with $h=\tau=1 / 32$ and 2 grids for $k=1$ with 
the same $h$ size, but using time steps $\tau_{1}=\sqrt{\tau}$. It is obvious that the higher order method produces solutions with smaller error. However, the relatively big difference is due to the relatively coarse grid in time, i.e. we should expect that the error size would be approximately the same for $k=0$ and $k=1$ at finer grids.

\section{REFERENCES}

[1] G. Akrivis and M. Crouzeix, Linearly implicit methods for nonlinear parabolic equations. Math. Comput. 73 (2004) 613-635.

[2] G. Akrivis and C. Makridakis, Galerkin time-stepping methods for nonlinear parabolic equations. ESAIM: M2AN 38 (2004) 261-289.

[3] G. Akrivis, M. Crouzeix and C. Makridakis, Implicit-explicit multistep finite element methods for nonlinear parabolic problems. Math. Comput. 67 (1998) 457-477.

[4] C. Chiu and N.J. Walkington, An ADI method for hysteric reaction-diffusion systems. SIAM J. Numer. Anal. 34 (1997) $1185-1206$.

[5] K. Chrysafinos and N.J. Walkington, Error estimates for the discontinuous Galerkin methods for parabolic equations. SIAM J. Numer. Anal. 44 (2006) 349-366.

[6] K. Chrysafinos and N.J. Walkington, Lagrangian and moving mesh methods for the convection diffusion equation. ESAIM: M2AN 42 (2008) 27-56.

[7] K. Chrysafinos and N.J. Walkington, Discontinous Galerkin approximations of the Stokes and Navier-Stokes problem. Math. Comput. 79 (2010) 2135-2167.

[8] P.G. Ciarlet, The finite element method for elliptic problems. SIAM Classics Appl. Math. (2002).

[9] M. Delfour, W. Hager and F. Trochu, Discontinuous Galerkin methods for ordinary differential equations. Math. Comput. 36 (1981) 455-473.

[10] S, Descombes and M. Ribot, Convergence of the Peaceman-Rachford approximation for reaction-diffusion systems. Numer. Math. 95 (2003) 503-525.

[11] K. Eriksson and C. Johnson, Adaptive finite element methods for parabolic problems. I. A linear model problem. SIAM J. Numer. Anal. 28 (1991) 43-77.

[12] K. Eriksson and C. Johnson, Adaptive finite element methods for parabolic problems. II. Optimal error estimates in $L_{\infty}\left(L^{2}\right)$ and $L_{\infty}\left(L_{\infty}\right)$. SIAM J. Numer. Anal. 32 (1995) 706-740.

[13] K. Ericksson and C. Johnson, Adaptive finite element methods for parabolic problems IV: Nonlinear problems. SIAM J. Numer. Anal. 32 (1995) 1729-1749.

[14] K. Eriksson, C. Johnson and V. Thomée, Time discretization of parabolic problems by the discontinuous Galerkin method. ESAIM: M2AN 29 (1985) 611-643.

[15] D. Estep and S. Larsson, The discontinuous Galerkin method for semilinear parabolic equations. ESAIM: M2AN 27 (1993) $35-54$.

[16] D. Estep, M. Larson and R. Williams, Estimating the error of numerical solutions of systems of reaction-diffusion equations. Mem. Amer. Math. Soc. 146 (2000) viii+109.

[17] L. Evans, Partial Differential Equations. AMS, Providence, RI (1998)

[18] P. Fife, Mathematical aspects of reacting and diffusing systems. Lect. Notes Biomath. 28 (1978).

[19] R. FitzHugh, Impulses and physiological states in theoretical models of nerve membrane. Biophys. J. 1 (1961) $445-466$.

[20] P. Franzone, P. Deflhard, B. Erdmann, J. Lang and L. Pavarino, Adaptivity in space and time for reaction-diffusion systems in electrocardiology. SIAM J. Sci. Comput. 28 (2006) 942-962.

[21] M.R. Garvie and J.M. Blowey, A reaction-diffusion system of $\lambda-\omega$ type. Part II: Numerical analysis. Eur. J. Appl. Math. 16 (2005) 621-646.

[22] M.R. Garvie and C. Trenchea, Finite element approximation of spatially extended predator interactions with the Holling type II functional response. Numer. Math. 107 (2008) 641-667.

[23] V. Girault and P.-A. Raviart, Finite Element Methods for Navier-Stokes. Springer-Verlag, New York (1986).

[24] M.D. Gunzburger, L.S. Hou and W. Zhu, Fully discrete finite element approximation of the forced Fisher equation. J. Math. Anal. Appl. 313 (2006) 419-440.

[25] E. Hansen and A. Ostermann, Dimension splitting for evolution equations. Numer. Math. 108 (2008) 557-570.

[26] S.P. Hastings, Some mathematical models from neurobiology. Amer. Math. Monthly 82 (1975) 881-895.

[27] W. Hundsdorfer and J. Verwer, Numerical solution for time-dependent advection-diffusion-reaction equations. Springer-Verlag, Berlin (2003).

[28] D. Jackson, Existence and regularity for the FitzHugh-Nagumo equations with inhomogeneous boundary conditions. Nonlinear Anal. Theory Methods Appl. 14 (1990) 201-216.

[29] D. Jackson, Error estimates for the semidiscrete Galerkin approximations of the FitzHugh-Nagumo equations. Appl. Math. Comput. 50 (1992) 93-114.

[30] P. Jamet, Galerkin-type approximations which are discontinuous in time for parabolic equations in a variable domain. SIAM J. Numer. Anal. 15 (1978) 912-928.

[31] C. Johnson, Numerical Solution of Partial Differential Equations by the Finite Element Method. Cambridge (1987).

[32] P. Lasaint and P.-A. Raviart, On a finite element method for solving the neutron transport equation, in Mathematical aspects of finite elements in partial differential equations, edited by C. de Boor. Academic Press, New York (1974) 89-123. 
[33] D. Meidner and B. Vexler, A priori error estimates for space-time finite element discretization of parabolic optimal control problems. Part I: Problems without control constraints. SIAM J. Control. Optim. 47 (2008) 1150-1177.

[34] C. Nagaiah, K. Kunisch and G. Plank, Numerical solution for optimal control problems of the reaction diffusion equations in cardiac electrophysiology. Comput. Optim. Appl. 49 (2011) 149-178.

[35] J.S. Nagumo, S. Arimoto and S. Yoshizawa, An active pulse transmission line simulating nerve axon. Proc. IRE 50 (1962) 2061-2070.

[36] C.S. Peskin, Partial Differential Equations in Biology. Courant Institute of Mathematical Sciences, New York (1975).

[37] M.E. Schoenbek, Boundary value problems for the FitzHugh-Nagumo equations. J. Differ. Equ. 30 (1978) $119-147$.

[38] R. Temam, Infinite Dimensional Dynamical Systems in Mechanics and Physics. Appl. Math. Sci. 68 (1997).

[39] C. Theodoropoulos, Y.-H. Qian and I.G. Kevrekidis, "Coarse" stability and bifurcation analysis using time-steppers: a reactiondiffusion example. Proc. Natl. Acad. Sci. USA 97 (2000) 9840-9843.

[40] V. Thomée, Galerkin finite element methods for parabolic problems. Spinger-Verlag, Berlin (1997).

[41] N.J. Walkington, Compactness properties of CG and DG schemes. SIAM J. Numer. Anal. 47 (2010) 4680-4710.

[42] E. Zeidler, Nonlinear functional analysis and its applications, in II/B Nonlinear monotone operators. Springer-Verlag, New York (1990). 